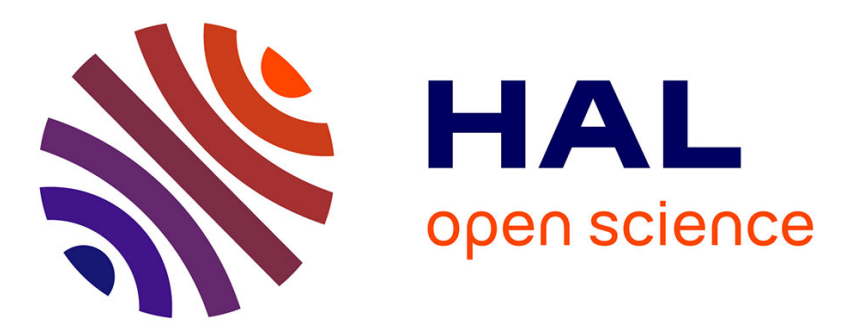

\title{
Optimal correction of distortion for high-angular-resolution images: Application to GeMS data.
}

\author{
A. Bernard, B. Neichel, L. Mugnier, T. Fusco
}

\section{- To cite this version:}

A. Bernard, B. Neichel, L. Mugnier, T. Fusco. Optimal correction of distortion for high-angularresolution images: Application to GeMS data.. Monthly Notices of the Royal Astronomical Society, 2018, 473 (2), pp.2590-2607. 10.1093/mnras/stx2517 . hal-01735857

\section{HAL Id: hal-01735857 \\ https://hal.science/hal-01735857}

Submitted on 16 Mar 2018

HAL is a multi-disciplinary open access archive for the deposit and dissemination of scientific research documents, whether they are published or not. The documents may come from teaching and research institutions in France or abroad, or from public or private research centers.
L'archive ouverte pluridisciplinaire HAL, est destinée au dépôt et à la diffusion de documents scientifiques de niveau recherche, publiés ou non, émanant des établissements d'enseignement et de recherche français ou étrangers, des laboratoires publics ou privés. 


\title{
Optimal correction of distortion for High Angular Resolution images. Application to GeMS data
}

\author{
A. Bernard ${ }^{1 \star} \dagger$, B. Neichel ${ }^{1}$, L. M. Mugnier ${ }^{2}$, T. Fusco ${ }^{2},{ }^{1}$ \\ ${ }^{1}$ Aix Marseille Université, CNRS, LAM (Laboratoire d'Astrophysique de Marseille) UMR 7326, 13388 Marseille, France \\ ${ }^{2}$ ONERA The French Aerospace Laboratory, F-92322 Chatillon, France
}

26 September 2017

\begin{abstract}
Whether is ground-based or space-based, any optical instrument suffers from some amount of optical geometric distortion. Recently, the diffraction-limited image quality afforded by space-based telescopes and by Adaptive Optics (AO) corrected instruments on ground basedtelescope, have increased the relative importance of the error terms induced by optical distortions. In particular, variable distortions present in Multi-Conjugated Adaptive Optics (MCAO) data are limiting the astrometry and photometry accuracy of such high resolution instruments. From there, the ability to deal with those phenomenon had become a critical issue for highprecision studies. We present in this paper an optimal method of distortion correction for high angular resolution images. Based on a prior-knowledge of the static distortion the method aims to correct the dynamical distortions specifically for each observation set and each frame. The method follows an inverse problem approach based on the work done by Gratadour et al. (2005) on image re-centering, and we aim to generalized it to any kind of distortion mode. The complete formalism of a Weighted Least Square minimization, as well as a detailed characterization of the error budget are presented. In particular we study the influence of different parameters such as the number of frames and the density of the field (sparse or crowed images), of the noise level, and of the aliasing effect. Finally, we show the first application of the method on real observations collected with the Gemini MCAO instrument, GeMS/GSAOI. The performance as well as the gain brought by this method are presented.
\end{abstract}

\section{CONTENTS}

1 Introduction

2 Data construction

2.1 Distortion modeling

2.2 Noise statistics

3 Algorithm description

3.1 Definition of a criterion

3.2 Practical implementation

3.3 Estimated parameters

3.4 Error criterion

4 Simulation results

4.1 Validation of the algorithm

4.2 Noise propagation

4.3 Aliasing effects

5 Application to GeMS data

5.1 Observation and data reduction

5.2 Construction of input catalogs

5.3 Distortion correction

6 Conclusion

\section{INTRODUCTION}

All optical instruments, whether is ground-based or space-based, suffer from some amount of optical geometric distortion. These distortions have multiple origins that induce different behavior. They may be static, resulting from unavoidable errors in the optics shape and placement or from imperfect fabrication of the detectors. For exemple, in the Wide Field Planetary Camera 2 (WFPC2) of the Hubble Space Telescope (HST), each of its four CCDs suffers from a distortion that reaches 5 pixels at the edge of the field (Anderson \& King (2003)). Distortions may be dynamic, due for exemple, to environmental parameters such as temperature variation: a typical illustration is the short term focus changes caused by small motions of the Hubble Space Telescope (HST) Optical Telescope Assembly (OTA) secondary mirror (referred to as OTA breathing in Hasan \& Bely (1994)). In Adaptive Optics (AO) corrected images from ground based telescopes, dynamical distortions may also be coming from residuals from the $\mathrm{AO}$ correction, or, in the case the $\mathrm{AO}$ system and the imaging camera are mounted at the Cassegrain focus of the telescope, from gravity flexure of the instrument as the telescope tracks (Cameron et al. (2009)).

The diffraction-limited image quality afforded by space-based telescopes and by AO corrected instruments on ground basedtelescopes, increases the relative importance of the error terms induced by these phenomena. A large number of studies evidence that the largest instrumental systematic that limits the astrometry accu- 


\section{A. Bernard}

racy in any optical system is the geometric distortion (Anderson \& King 2003; Cameron et al. 2009; Trippe et al. 2010; Fritz et al. 2010). As the goal of astrometry is to measure the position of target stars over many epochs, it is usually done on combined objects frames with varying pointing positions (technique of dithering), and varying epochs. The knowledge of the distortion is then necessary to place stellar positions in a globally correct reference frame. Distortion can also seriously limit photometric accuracy on stack images that are essential to photometer the faintest objects (Bernard et al. (2016)). Indeed, if it is stated that the angular resolution on stacked images is always worse that the angular resolution on a single frame, stacking images uncorrected for distortion amplified this degradation proportionally to the amount of present distortion. For exemple, in the case of images collected with the Gemini Multi-Conjugated Adaptive Optics System (GeMS), the final angular resolution on a stack of images uncorrected for distortion can reach almost twice the typical angular resolution on a single frame (which is about 90 mas).

The importance of distortion effects in high angular resolution imaging has led researchers to develop a number of strategies to mitigate their impact. The most notable method is the self calibration developed by Anderson \& King (2003) to derive with high accuracy (to $\sim 1$ milliarcsecond (mas)) the distortion of the HST WFPC2. The method consists in using the instrument to calibrate itself. The standard way to do this is to take multiple observations of the same field at different offsets and orientations, so that the same patch of sky is imaged on different regions of the detector. Observed positions for all the stars in all the images are measured, and a single distortion model is found. This method dispenses the need for an external reference frame, as the distortion-free reference positions are derived from the observations themselves. However, this requires a large set of on-sky data with large translations at many orientations to constrain all high-order modes of distortion. For exemple, the solution derived by Anderson \& King (2003) required 80 exposures and 4000 stars per images. This kind of calibration, which cannot be implemented for each observation, suppose that the distortion is stable in time. It is well adapted to space-based telescopes where the weightless environment means that telescope flexure does not lead to large changes in the distortion solution. Thus, this method has next been used for numerous HST cameras: the Advanced Camera for Survey High Resolution Channel (ACS/HRC) with a precision of 0.25 mas (Anderson \& King (2004)), the UVIS channel of the WFPC3 with a precision of 1 mas (see Bellini \& Bedin (2009)) and the ACS Wide Field Channel (WFC) with a precision of 1 mas (Ubeda \& Kozhurina-Platais (2013)). Later, Anderson et al. (2006) adapted this solution for ground-based telescopes by calibrating the Wide Imager Field (WIF) at the European Southern Observatory 2.2 meter telescope with a precision of 7 mas (on each coordinate). Following the same process, a distortion solution was derived for the High Acuity Wide field K-band Imager (HAWK-I) at the Nasmyth focus of Unit Telescope 4 / Very Large Telescope (UT4/VLT) ESO 8 meter telescope with a precision of 3 mas (Libralato et al. (2014)), and for the Large Binocular Cameras-blue, at the prime focus of the Large Binocular Telescope with a precision of 15 mas (Bellini et al. (2010)).

Another way to solve for the distortion present in an instrument is to observe a field where we have prior knowledge of the reference positions of all the stars in a distortion-free system. Distortion would then show itself immediately as the residuals between the observed and the reference positions of stars. Reference positions can be derived from external astrometric reference frames, such as astrometric catalogs (UCAC-2, GSC-2 or 2MASS) or HST observations (accurately corrected from distortion by self-calibration as mentioned previously) as it was done by Yelda et al. (2010) and Service et al. (2016) to calibrate the Near-InfraRed Camera 2 (NIRC2) on the Keck II 10 meters telescope. The cross-calibration might also be done using datasets from other wavelength regimes. A nice example is given by Reid \& Menten (2007) who used precise Very-LongBaseline Interferometry (VLBI) astrometry of $\mathrm{SiO}$ maser stars in order to define an astrometric reference frame in K-band images of the Galactic center. The major advantage of this method is that a much smaller set of on-sky data is needed as compared to the self-calibration method. The final error on the distortion calibration is then set by the residual distortion in the distortion-free external reference used.

The difficulty to calibrate distortion on ground-based telescopes is due to the variability of the distortions. Some are quite stable, as the NIRC2 on the Keck II telescope which system was stable over the period from 2007-2010 (Yelda et al. (2010)), but have to be calibrated again after modifications in the system such as realignment (Service et al. (2016)), and some are varying in time. Thus, the solution derived for the WIF/ESO, varied of about 100 mas in 3 years and the one derived for HAWK-I/VLT varied of about 3 mas on 3 months scale. This phenomenon is particularly presents in MultiConjugate Adaptive Optics (MCAO) systems where the distortion may vary during one night (Neichel et al. (2014b), Massari et al. (2016)). The most likely hypothesis on the origins of these variable distortions is that they might be introduced by the deformable mirrors conjugated in altitude. In this context, several authors have performed a relative correction of the distortion based on a mastercoordinate-frame created by averaging the position of each star over all frames and considered as a reference. With this method, the correction can adapt to each set of data and each single frame, but the reference is not absolute. The measurement of the relative astrometric precision achieved with this method is then calculated as the Root-Mean-Square (RMS) deviation of the positions through the different frames. The relative astrometric precision ranges from 1.2 to 2.8 mas for MCAO Demonstrator MAD (Meyer et al. (2011)) and reaches 0.4 mas on GeMS/GSAOI data Neichel et al. (2014b).

Finally, some in-lab methods are developed to characterize the instrument distortion with dedicated measurements in the laboratory or at the telescope. One example is the "north-south test" used for the spectro-imager SINFONI at the VLT (e.g. Abuter et al. (2006)). This method uses devices that illuminate the detectors with welldefined images or light patterns. Comparing the theoretical with the observed images allows for a description of the distortion. Another exemple is the use of a calibration mask located in the focal plane of the imager. Such a mask could be a regular pattern of holes in an opaque material. The precision of the holes positions in the calibration mask determines the accuracy of the distortion calibration. For exemple, distortions in NIRC2 were initially characterized using illuminated pinhole masks (Cameron \& Kulkarni (2007)). However, the residual distortion in those solutions was larger than the distortion solution produced using on-sky method (Service et al. (2016)).

In this paper, we present a new method of distortion correction based on a prior-knowledge of the static distortion as a starting point to correct the residual distortion (referred hereafter as dynamical distortion). Thus, the correction to apply is calculated specifically for each frame of each observation set. Based on a Weighted Least Squares (WLS) minimization, this method has the particularity to provide an estimation of both a distortion-free reference and a distortion solution associated to each frame with a limited noise propagation thanks to the weighted property of the minimization. 
Both estimated parameters can be used independently depending on the scientific aim of the study: for exemple, absolute astrometry requires a good estimation of the distortion-free reference, whereas a good estimation of the distortion solution associated to each frame is needed for relative astrometry applications (that require an good ability to place stellar positions in a common reference frame) and for photometry studies (that require a good ability to stack the images). The performance of these estimations depends on the nature of the data (crowded or sparse fields, number of frames, noise level) and are detailed in Section 4. The paper is constructed as follow : In Section 2, we expose hypothesis on the data construction and on the noise statistical model. Section 3 is dedicated to the description of the minimization process, and of the algorithm implementation. In Section 4, we describe the validation of the proposed method using simulated data, and Section 5 shows its first application on on-sky data. The performance as well as the gain brought by this method are presented in this section.

\section{DATA CONSTRUCTION}

The distortion correction method presented in this paper follows an inverse problem approach based on the work developed by D. Gratadour and L. Mugnier in Gratadour et al. (2005). Their original work was focusing on isoplanatic image recentering while we generalize it to any kind of distortion in the field. The inverse problem approach requires the modeling of the data formation process (which is called the direct problem), in order to take it into account during the inversion. The direct problem provides the calculation of the data associated to an known object, it is thus used to build simulated data. This section is dedicated to the description of this direct problem, including the modeling of the distortions and the description of the statistical noise properties considered.

\subsection{Distortion modeling}

The object of interest considered here is the distortion-free reference frame $I^{r e f}$ that would be delivered by a distortion-free instrument. The associated data is then the image, noted $I^{\text {data }}$, delivered by a real instrument. The operation that connects the object to the data is a distortion function noted $D$, that affects coordinates:

$I^{\text {data }}[.,]=.I^{r e f}[D(.,)$.

This expression adapts to each frame $i$, with its associated distortion function. It can apply to any basis: from the most exhaustive one, the pixels themselves, to a subset of it such as a set of as local positions computed on specific point like source objects. Hereafter, we consider a basis of stars positions, where the position of star $j$ in frame $i$ is noted $X_{i, j}^{\text {data }}$. Following the previous reasoning, any star position measured in the data, $X_{i, j}^{\text {data }}$, can be described as the position of the star $j$ in the reference frame $X_{j}^{r e f}$, on which the distortion function $D_{i}$ is applied.

$X_{i, j}^{\text {data }}=D_{i}\left(X_{j}^{r e f}\right)$

From here, different functions can be used to describe the distortion. Most commonly, 2D polynomials up to about $3 \mathrm{rd}$ or 5 th order, depending on the authors, are used as distortion models. For exemple, Anderson \& King (2003) used a 3rd order polynomial fitting to correct distortion on the HST/WFPC2, while Libralato et al. (2014) used a 5th order polynomial fitting for the HAWK-I calibration. The NIRC2 camera at the W.M. Keck Observatory, was successively corrected for distortion using a polynomial fitting (see Ghez et al. (2008), and Lu et al. (2009)), a bivariate B-spline fitting (Yelda et al. (2010)) and more recently a 2D Legendre polynomials fitting (Service et al. (2016)). The last authors compare the efficiency of different models: 2D cartesian polynomials, bivariate B-spline and 2D Legendre polynomial. They conclude that the 2D Legendre polynomial basis provides a faster convergence and lower residuals. Following this study, we choose the 2D Legendre polynomial basis, which combines several advantages, to define our distortion model. First, the 2D Legendre polynomial basis is an orthogonal basis (e.g. Dunkl \& Xu (2014), Ye et al. (2014) ) defined on a square, thus well adapted to describe the distortion on square images. Then, the ascending polynomial degree organization of the basis is convenient to characterize the distortion using a limited number of modes. Each Legendre polynomial $P_{n}(x)$ is an $n$ th-degree polynomial and may be expressed in one dimension as follows:

$P_{n}(x)=\frac{1}{2^{n}} \sum_{k=0}^{n}\left(\begin{array}{l}n \\ k\end{array}\right)^{2}(x-1)^{n-k}(x+1)^{k}, x \in[-1 ; 1]$

In addition, we choose to normalize the polynomials so that each mode contains the same amount of distortion. The final 2D distortion basis $B$ is defined as follows :

$B_{N_{\text {modes }}}(x, y)=\left(\begin{array}{c}L_{0}(x) \cdot L_{0}(y) \\ L_{1}(x) \cdot L_{0}(y) \\ L_{0}(x) \cdot L_{1}(y) \\ L_{2}(x) \cdot L_{0}(y) \\ L_{1}(x) \cdot L_{1}(y) \\ L_{0}(x) \cdot L_{2}(y) \\ \cdots\end{array}\right)=\left(\begin{array}{c}\frac{1}{2} \\ \frac{\sqrt{3}}{2} x \\ \frac{\sqrt{3}}{2} y \\ \frac{\sqrt{5}}{4}\left(x^{2}-1\right) \\ \frac{3}{2} x y \\ \frac{\sqrt{5}}{4}\left(y^{2}-1\right) \\ \cdots\end{array}\right)(x, y) \in[-1 ; 1]^{2}$

where:

$L_{n}$ is the $n$th degree normalized Legendre polynomial: $L_{n}=\frac{P_{n}}{\left\|P_{n}\right\|}$ with $\left\|P_{n}\right\|^{2}=\frac{2}{2 n+1}$

$N_{\text {modes }}$ is the number of modes considered. It determines the dimension of the vector $B_{N_{\text {modes }}}$.

Let us recall that the 2D Legendre polynomial basis conserves the orthogonality property with respect to the inner product $\langle.,$.$\rangle de-$ fined on $[-1,1]^{2}$ as follows:

$\langle f, g\rangle=\int_{-1}^{1} \int_{-1}^{1} f(x, y) \cdot g(x, y) d x d y$

Hereafter, the distortion modes are referred to using their index mode $(m)$ defined as:

$m(k, l)=l(l+1) / 2+k \quad$ and $b_{m}(x, y)=L_{l-k}(x) * L_{k}(y)$

where $0 \leq k \leq l \in \mathbb{N}$ and $0 \leq m \leq N_{\text {modes }}$.

Figure 1 shows the displacements induced by the three first modes of the distortion basis $B$, described by the $2 \mathrm{D}$ normed Legendre polynomials. The displacements are applied to the $x$-coordinates. The left panel shows the reference grid without any distortion. 

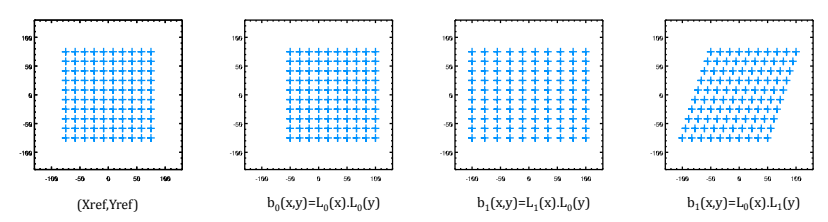

Figure 1. Displacements induced by the three first modes of the distortion basis $B$, described by the $2 \mathrm{D}$ normalized Legendre polynomials. The displacements are applied to the $x$-coordinates. The left panel shows the reference grid without any distortion.

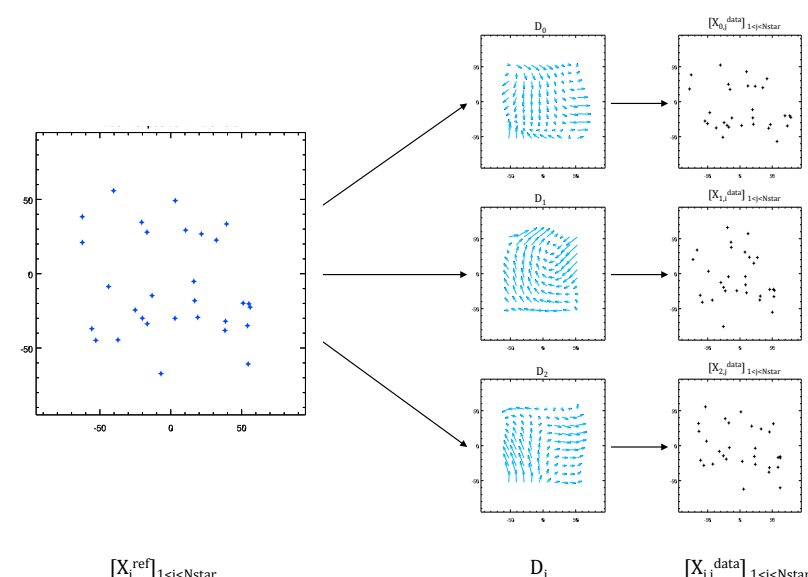

Figure 2. Schematic diagram of the distortion application on different images. Left panel: reference postions $\left[X^{r e f}\right]_{1 \leq j \leq N_{\text {star }} \text {. Middle panel: set }}$ of distortion maps, $D_{i}$ (where $i=0,1,2$ ). Right panel: resulting positions after application of the associated distortion map $\left[X^{\text {data }}{ }_{i, j}\right]_{1 \leq j \leq N_{\text {st ar }}}$.

Finally, the position of a star $j$, on which a distortion $D_{i}$ is applied can be written as follows:

$X_{i, j}=D_{i}\left[X_{j}^{r e f}\right]=\left\{\begin{array}{c}x_{j}^{r e f}+\sum_{l=0}^{d} \sum_{k=0}^{l} a_{i, m, x} b_{m}\left(x_{j}^{r e f}, y_{j}^{r e f}\right) \\ y_{j}^{r e f}+\sum_{l=0}^{d} \sum_{k=0}^{l} a_{i, m, y} b_{m}\left(x_{j}^{r e f}, y_{j}^{r e f}\right)\end{array}\right.$

where:

$d$ is the maximal polynomial order considered. Note that $N_{\text {modes }}=(d+1)(d+2) / 2$.

For each image, the set of decomposition coefficients, $\left(a_{i, m, y / x}\right)_{0 \leq m \leq N_{\text {modes }}}$ is noted as $A_{i}$ and referred to as the distortion coefficients. The application of the distortion on different images is shown in Figure 2. The left panel shows the reference positions $\left[X^{r e f}\right]_{1 \leq j \leq N_{\text {star }}}$, with $N_{\text {star }}$ the number of reference sources, the middle panel shows the displacement induced by a set of distortion maps, $D_{i}$ (with $i=0,1,2$ ) and the right panel shows the distorted positions associated to each distortion map $\left[X^{\text {data }}{ }_{i, j}\right]_{1 \leq j \leq N_{\text {star }}}$.

\subsection{Noise statistics}

The statistical properties of the noise that we should consider to build the data model must be consistent with the noise present in measurement of star positions. We assume here a noise that follows a Gaussian white distribution (Gaussian White Noise). This hypothesis is motivated by the following statements: first, the images are contaminated by two main type of noise: the detector noise, which follow a Gaussian distribution, and the photon noise which follows a Poisson distribution. However, it has been shown thats for a high level of flux, a Poisson distribution converge towards a Gaussian distribution (see e.g. Mugnier et al. (2004)). Then, the position's measurements combine informations of many pixels. Following the central limit theorem, the noise present in these measurements tends toward a normal distribution. As the noise present in each measurement is independent, we can consider with a very good approximation that the noise present in the data is white and Gaussian, with a variance noted $\sigma_{\text {meas }}^{2}$ that may depend on image number $i$ and star number $j$. The final data model, a.k.a. direct model, is thus:

$X_{i, j}=D_{i}\left[X_{j}^{r e f}\right]=\left\{\begin{array}{c}x_{j}^{r e f}+\sum_{l=0}^{d} \sum_{k=0}^{l} a_{i, m, x} L_{l-k}\left(x_{j}^{r e f}\right) L_{k}\left(y_{j}^{r e f}\right)+n_{i, j, x} \\ y_{j}^{r e f}+\sum_{l=0}^{d} \sum_{k=0}^{l} a_{i, m, y} L_{l-k}\left(x_{j}^{r e f}\right) L_{k}\left(y_{j}^{r e f}\right)+n_{i, j, y}\end{array}\right.$

where $n_{i, j, x}$ and $n_{i, j, y}$ are independent Gaussian noises, with variance $\sigma_{\text {meas }, i, j}$, of the position measurements.

\section{ALGORITHM DESCRIPTION}

In the previous section we defined the direct model. To solve the distortion problem, we now aim to inverse this model through the minimization of a criterion. In this section, we define the final criterion to be minimized and describe the implementation of the resulting algorithm. Then, we present the estimated parameters utilisations, and the error criteria associated.

\subsection{Definition of a criterion}

The final direct model is described by Equation (8). In this equation, both the distortion coefficients $A_{i}$, and the reference positions $X_{j}^{r e f}$, are unknown. The proposed method aims to perform a joint estimation of these two parameters for all the stars $j$ (with $j \leq N_{\text {star }}$ and $N_{\text {star }}$ the number of stars considered) and all the frames $i$ (with $i \leq N_{i m}$ and $N_{i m}$ the number of frames). We introduce here the following notations: tions

. $\left[X^{r e f}\right]=\left[X_{j}^{r e f}\right]_{1 \leq j \leq N_{\text {star }}}$, the whole set of reference posi. $[A]=\left[A_{i}\right]_{1 \leq i \leq N_{i m}}$, the whole set of distortion coefficients.

Considering the assumptions made in Section 2.2 on the noise statistical distribution, the Maximum Likelihood estimation of these unknowns boils down to the Weighted Least Square minimization of the following criterion (e.g. Mugnier (2008)):

$J\left(\left[X^{r e f}\right] ;[A]\right)=\left\{\begin{array}{c}\sum_{j=1}^{N_{s t a r}} \sum_{i=1}^{N_{i m}} w_{i, j}\left|X_{i, j}^{\text {data }}-\left(x_{j}^{r e f}+\sum_{l=0}^{d} \sum_{k=0}^{l} a_{i, m, x} L_{l-k}\left(x_{j}^{r e f}\right) L_{k}\left(y_{j}^{r e f}\right)\right)\right|^{2} \\ N_{s \text { sar }} N_{i m} \sum_{i=1} w_{i, j}\left|y_{i, j}^{d a t a}-\left(y_{j}^{r e f}+\sum_{l=0}^{d} \sum_{k=0}^{l} a_{i, m, y} L_{l-k}\left(x_{j}^{r e f}\right) L_{k}\left(y_{j}^{r e f}\right)\right)\right|^{2} \\ \quad j=1\end{array}\right.$

where $w_{i, j}$ is a weighing coefficient that can be used to quadratically weight the impact of each data in the criterion according to their 
Signal to Noise Ratio (SNR):

$w_{i, j}=\frac{1}{\sigma_{\text {meas }, i, j}^{2}}$

Note that the number of available reference sources governs the maximum order of coordinate transformations: A 1st order polynomial transform with six parameters requires at least three reference sources, i.e., six coordinates. A 2nd-order transform (12 parameters) requires at least six reference sources, and so on. In short, we must have $N_{\text {star }} \geq N_{\text {modes }}$.

\subsection{Practical implementation}

This section details the practical implementation for the minimization of the criterion defined in Equation (9). As mentioned previously, the aim of the algorithm is to perform a joint estimation of both:

$$
\begin{aligned}
& \cdot \text { the reference positions of all the stars }\left[X_{j}^{r e f}\right]_{1 \leq j \leq N_{\text {star }}} \text { noted } \\
& {\left[X^{r e f}\right] \text {; }} \\
& \cdot \text { the distortion coefficients of all frames }\left[A_{i}\right]_{1 \leq i \leq N_{i m}} \text { noted }
\end{aligned}
$$
[A]

In practice, this criterion is minimized in an alternate fashion with respect to one set of variables, while the second is set. We detail hereafter the two steps included in one iteration of the minimization. For convenience, we detail the equation corresponding to the $x$-coordinates only, but the expressions can transposed to the $y$-coordinates. The estimation of a parameter $p$ is noted $\widehat{p}$.

The first step of the alternate estimation is to find $\left[X^{r e f}\right]$ for the current value of $[A]$. For a given $[A]$, it is easy to realize that criterion $J\left(\left[X^{r e f}\right] ;[A]\right)$ of Equation (9) can be written as a sum of $N_{\text {star }}$ independent terms:

$$
J\left(\left[X^{r e f}\right] ;[A]\right)=\sum_{j=1}^{N_{\text {star }}} J_{1}\left(X_{j}^{r e f},[A]\right)
$$

where:

$J_{1}\left(X_{j}^{r e f} ;[A]\right)=\sum_{i=1}^{N_{i m}} w_{i, j}\left|X_{i, j}^{\text {data }}-\left(x_{j}^{r e f}+\sum_{l=0}^{d} \sum_{k=0}^{l} a_{i, m, x} L_{l-k}\left(x_{j}^{r e f}\right) L_{k}\left(y_{j}^{r e f}\right)\right)\right|^{2}$

Hence, the estimation of the reference positions can be done independently for each star $j$ and is obtained as:

$\widehat{X}_{j}^{\text {ref }}=\underset{X_{j}^{\text {ref }}}{\arg \min _{j}}\left[J_{1}\left(X_{j}^{\text {ref }} ;[A]\right)\right]$

The same reasoning can be applied for the second step. For a given $\left[X^{r e f}\right]$, criterion $J\left(\left[X^{r e f}\right] ;[A]\right)$ of Equation (9) can be written as a sum of $N_{i m}$ independent terms:

$$
J\left(\left[X^{r e f}\right] ;[A]\right)=\sum_{i=1}^{N_{i m}} J_{2}\left(\left[X^{r e f}\right], A_{i}\right)
$$

where:

$J_{2}\left(A_{i} ;\left[X^{r e f}\right]\right)=\sum_{j=1}^{N_{s t a r}} w_{i, j}\left|X_{i, j}^{\text {data }}-\left(x_{j}^{r e f}+\sum_{l=0}^{d} \sum_{k=0}^{l} a_{i, m, x} L_{l-k}\left(x_{j}^{r e f}\right) L_{k}\left(y_{j}^{r e f}\right)\right)\right|^{2}$

The estimation of the distortion coefficients can thus be done independently for each frame $i$ :

$\widehat{A_{i}}=\arg \min _{A_{i}}\left[J_{2}\left(A_{i} ;\left[X^{r e f}\right]\right)\right]$
These two steps are performed sequentially until a stopping condition relative to the convergence is reached. For exemple, we choose to stop the convergence when the difference of the estimates between two iterations is lower than $10^{-5}$ :

$C_{A}^{(k)}=\frac{\left|[\widehat{A}]^{(k-1)}-[\widehat{A}]^{(k)}\right|}{[\widehat{A}]^{(k)}} \leq 10^{-5}$

and,

$C_{X^{r e f}}^{(k)}=\frac{\left|\left[\widehat{X}^{r e f}\right]^{(k-1)}-\left[\widehat{X}^{r e f}\right]^{(k)}\right|}{\left[\widehat{X}^{r e f}\right]^{(k)}} \leq 10^{-5}$

where $\widehat{p}^{(k)}$ is the estimation of parameter $p$ at the $k$-th iteration. To summarize, a bloc diagram of the algorithm is presented hereafter (Figure 3).

When both parameters, $[\widehat{A}]$ and $\left[\widehat{X}^{r e f}\right]$ are estimated, both of them can be useful and their use depends on the final physical objective. The reader's attention is here drawn to one important point: the parameters are estimated regarding the data set provided. If the instrument suffers from static distortions, for exemple a global shift, then the data collected with this instrument are all globally shifted regarding to the celestial coordinates. The same holds true if the instrument suffers from a global scale factor: the data collected with this instrument are all globally scaled. These global modes cannot be measured unless a priori knowledge of the static distortion is available from a previous calibration. In the latter case, the proposed method derives distortion solutions calibrated for each observation set and for each frame, that varies around the static distortion provided. In the following, we address this configuration and consider a priori knowledge of the instrument's static distortion. In practice, the static distortion is implemented as initial parameters for the distortion coefficients $[A]^{0}$.

\subsection{Estimated parameters}

Both estimated parameters $[\widehat{A}]$ and $\left[\widehat{X}^{r e f}\right]$, can be used independently depending on the scientific aim of the study at hand.

In the case of absolute astrometry studies, the information required is simply the estimate of the reference positions [ $\left.\widehat{X}^{\text {ref }}\right]$, obtained (12) as described above.

In the case of relative astrometry or photometry studies, an additional step is needed, namely to correct the different frames from the distortion. This, aiming to place stellar positions in a relative global reference frame or to stack the images. In that case, the parameters of interest are the estimated distortion coefficients $[\widehat{A}]$, of all frames. The correction of the distortion may apply to the star positons only, or to the entire image thanks to an interpolation process. In the latter case we want to correct star positions only, the corrected position of each star $j$, in the frame $i$ is noted $X_{i, j}$ corr and calculated independently as follow:

$\widehat{X}_{i, j}^{\text {corr }}=\arg \min _{X_{i, j}^{\text {corr }}}\left[J_{3}\left(X_{i, j}^{\text {corr }} ;[\widehat{A}]\right)\right]$

where:

$J_{3}\left(X_{i, j}^{\text {corr }} ;[\widehat{A}]\right)=\left|X_{i, j}^{\text {data }}-\left(x_{i, j}^{\text {corr }}+\sum_{l=0}^{d} \sum_{k=0}^{l} a_{i, m, x} L_{l-k}\left(x_{i, j}^{\text {corr }}\right) L_{k}\left(y_{i, j}^{\text {corr }}\right)\right)\right|^{2}$

and equivalent for the $y$-coordinates. 


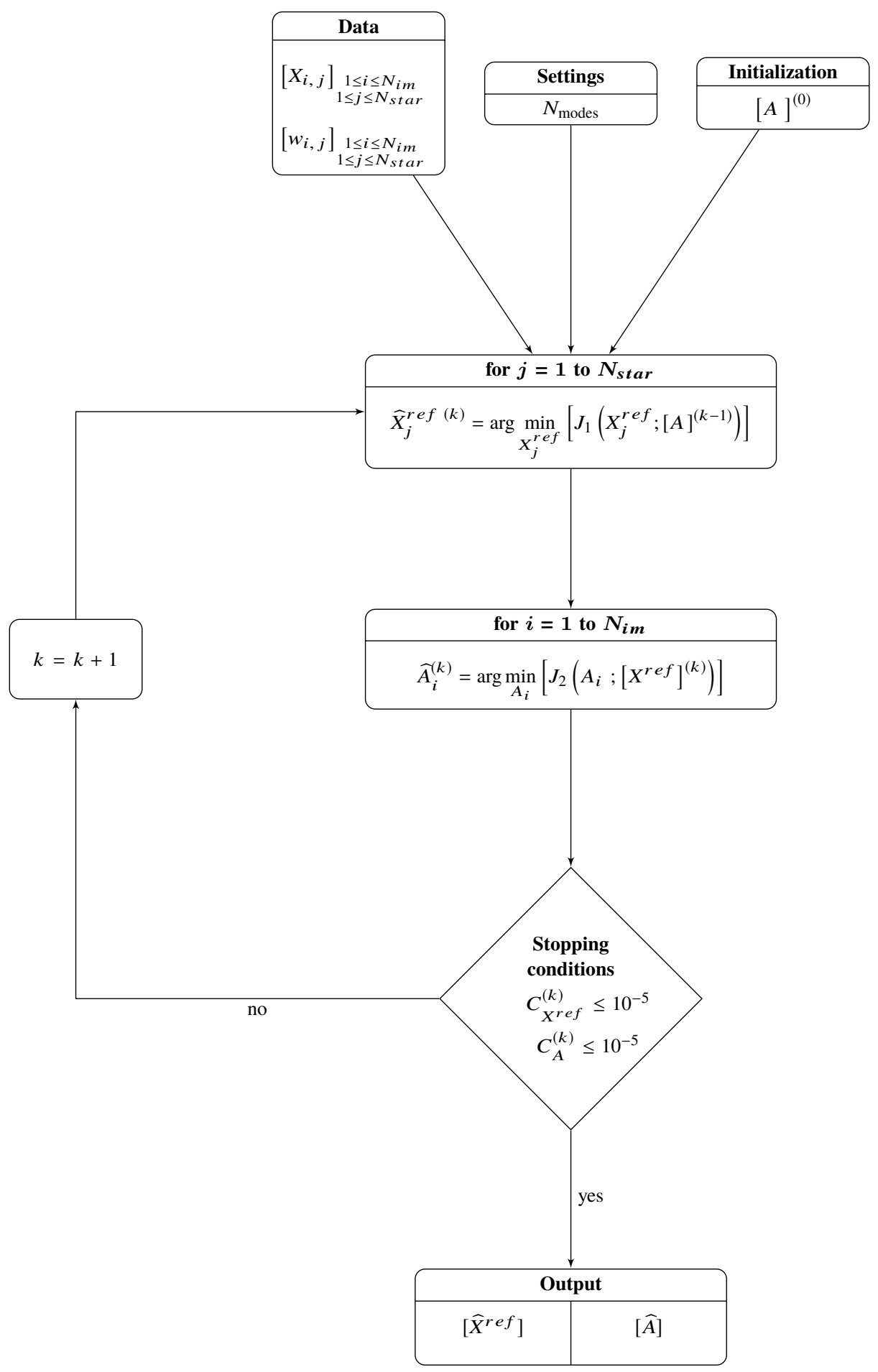

Figure 3. Bloc diagram of the minimization algorithm of Equation (9) multi-variable $J$ criterion. 
Note that $J_{1}$ is the sum of $N_{i m} J_{3}$-like terms, so that $\widehat{X}_{i, j}^{\text {corr }}$ is the reference position of star $j$ as predicted by the distortion map $\widehat{A}_{i}$ alone. Whereas $\widehat{X}_{j}^{r e f}$ is the reference position of star $j$ that best fits all the estimated distortion maps.

The effect of the algorithm on one particular star $j_{0}$, is illustrated by Figure 4: the left panel shows the set of $\left[X_{i, j_{0}}^{\text {data }}\right]_{1 \leq i \leq N i m}$ before distortion correction (black crosses), as well as the true reference position $X_{j_{0}}^{r e f}$ (red cross). The right panel shows the set of positions corrected from distortion $\left[\widehat{X}_{i, j_{0}}^{\text {corr }}\right]_{1 \leq i \leq N i m}$ (black crosses), using the estimated distortion coefficient $[\widehat{A}]$. Bottom panel is a zoom of right panel: it additionally shows the estimated reference position $\widehat{X}_{j_{0}}^{r e f}$ (blue cross) and the averaged corrected positions on all images (orange cross). Note that these two positions match each other: $\operatorname{avg}_{i}\left(\widehat{X}_{i, j_{0}}^{\text {corr }}\right) \simeq \widehat{X}_{j_{0}}^{r e f}$. This result is expected from the definition of the $J_{1}$ and $J_{3}$ criteria and it can easily be demonstrated in the case of linear transformations (i.e. $d=0$ ). In practice, we observe that this equality is verified for any transformation order, within a few $10^{-3}$ pixels. The algorithm performance can then be quantified using two error criteria illustrated on the bottom panel : a bias represented as a blue arrow; and a standard deviation shown as a black arrow. Both error criteria are described analytically in the next section (3.4).

\subsection{Error criterion}

As mentioned previously, the performance of the algorithm, is quantified by two criteria that we define as a bias and a standard deviation as follows:

- The bias quantifies the ability to estimate the reference positions. It is defined for each star by the distance between the estimated reference position, $\widehat{X}_{j}^{r e f}$ and the reference position, $X_{j}^{r e f}$. It is noted $b_{j}$ hereafter.

$b_{j}=\widehat{X}_{j}^{r e f}-X_{j}^{r e f}$

This error is shown as a blue arrow in Figure 4 and can be quadratically averaged on all the stars:

$b=\sqrt{\frac{1}{N_{\text {star }}} \sum_{j=1}^{N_{\text {star }}}\left|\widehat{X}_{j}^{\text {ref }}-X_{j}^{\text {ref }}\right|^{2}}$

- The standard deviation, which quantifies the algorithm ability to place every stellar position in a global reference frame or to stack the images. It is the standard deviation of each star's corrected position through the stack of images and it is noted $\sigma_{j}$.

$\sigma_{j}=\sqrt{\frac{1}{N_{i m}} \sum_{i=1}^{N_{i m}}\left|\widehat{X}_{i, j}^{c o r r}-\operatorname{avg}\left(\widehat{X}_{i, j}^{\text {corr }}\right)\right|^{2}}$

This error is represented as a black arrow on Figure 4. Note that this error is lower bounded by the measurement error $\sigma_{\text {meas, } j}$ of each star $j$, which is the fundamental limit of the astrometric precision in the case of a perfect optical system:

$\sigma_{\text {meas, } j} \leq \sigma_{j}$

The information given by this last criterion is very local in the field. It is calculated at the position of the reference sources used in the

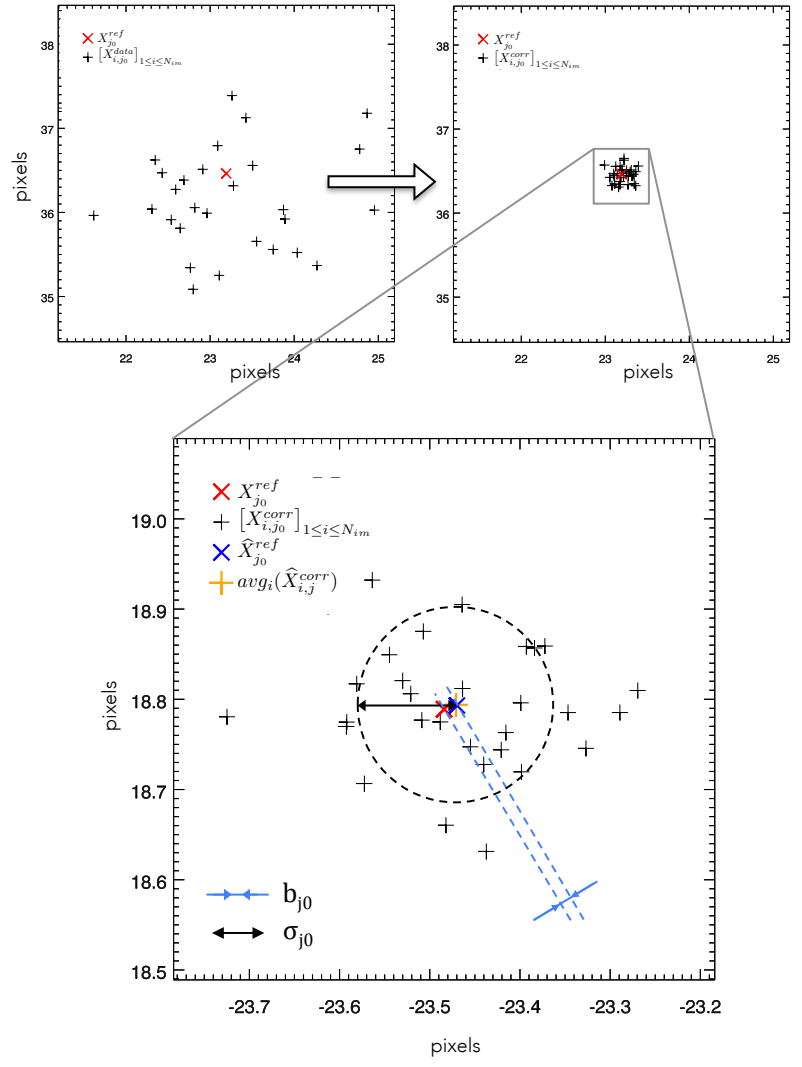

Figure 4. Illustration of the algorithm effect on the star $j_{0}$, in the case of $N i m=30$. Left panel: set of $\left[X_{i, j_{0}}^{\text {data }}\right]_{1 \leq i \leq N i m}$ before distortion correction (black crosses) and true reference position $X_{j_{0}}^{r e f}$ (red cross). Right panel: set of positions corrected from distortion $\left[\widehat{X}_{i, j_{0}}^{\text {corr }}\right]_{1 \leq i \leq N i m}$ (black crosses). Bottom panel: zoom of right panel. It additionally shows the estimated reference position $\widehat{X}_{j_{0}}^{r e f}$ (blue cross) and the averaged corrected positions on all images (orange cross). Note that $a v g_{i}\left(\widehat{X}_{i, j_{0}}^{c o r r}\right) \simeq \widehat{X}_{j_{0}}^{r e f}$. The two error criteria used to quantify the algorithm performance are also illustrated on bottom panel. The first one is a bias: the distance between the true reference position, $X_{j_{0}}^{r e f}$ (red cross) and the estimated reference position, $\widehat{X}_{j_{0}}^{r e f}$ (blue cross). It is noted $b_{j_{0}}$ and represented in blue arrow. The second one is the standard deviation of the corrected positions (schematized in black arrow) is noted $\sigma_{j_{0}}$. Both error criterion are described in further details in section 3.4.

minimization. In order to evaluate the ability to stack the whole image, this error criterion is generalized to stars that are not used in the resolution and that are distributed as a regular grid in the field. Hereafter, this generalized criterion is noted $\sigma$, the number of stars considered in the regular grid is noted $N_{\text {star, grid }}$ and the stars included in the grid are indexed $j^{\prime}$ :

$$
\sigma=\sqrt{\frac{1}{N_{\text {star, grid }}} \sum_{j^{\prime}=1}^{N_{\text {star, grid }}} \sigma_{j^{\prime}}^{2}}
$$




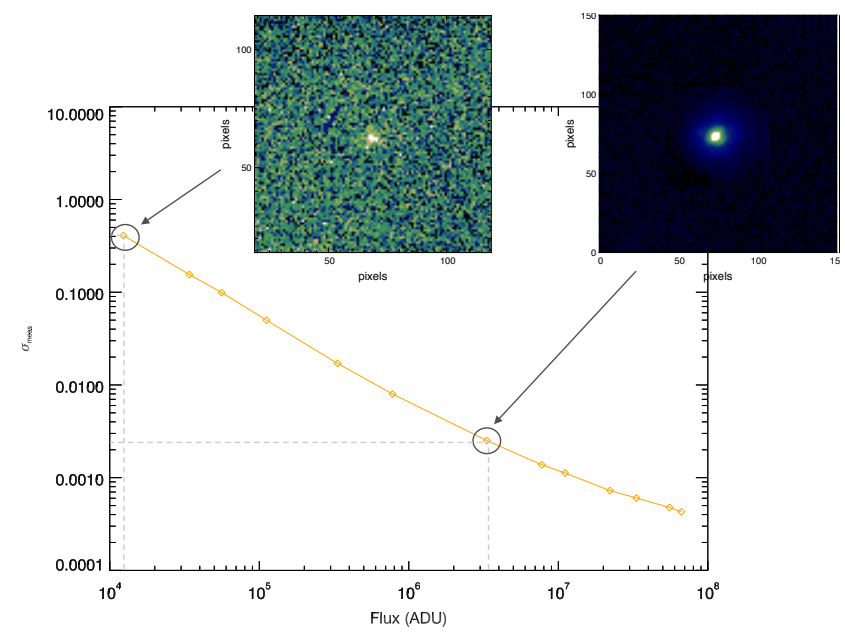

Figure 5. Error on position measurements versus the flux on typical GeMS/GSAOI data. Are also represented, one star fitted with an error of $\sigma_{\text {meas }}=0.14$ pixels on its positions and one star fitted with an error of $\sigma_{\text {meas }}=0.002$ pixels on its positions, with a Levenberg-Marquart fitting tool. The last one corresponds to a 18 magnitude in $K_{\mathrm{s}}$ band for a $60 \mathrm{~s}$ exposure.

\section{SIMULATION RESULTS}

This section aims at validating the algorithm and investigating its performance using simulated data representative of those collected with the GeMS/GSAOI systems. The data are simulated according to the direct model described in Section 2 and the noise introduced in the position measurements is a Gaussian white noise (as explained in Section 2.2), with a variance $\sigma_{\text {meas }}^{2}$. In typical GeMS data, the error on position measurements ranged between 0.002 pixels and 0.14 pixels $(0.02$ mas and 2.8 mas respectively), mostly depending on the magnitude of the objects. For example, we show in Figure 5 two stars, one fitted with an error of $\sigma_{\text {meas }}=0.002$ pixel, and one fitted with an error of $\sigma_{\text {meas }}=0.14$ pixel, in typical GeMS/GSAOI data with a Levenberg-Marquart fitting tool. The last one represents the faintest stars detectable in a single frame, corresponding to a 18 magnitude in $K_{\mathrm{S}}$ band for a $60 \mathrm{~s}$ exposure.

For each simulation, the performance are evaluated regarding the two error criteria defined in Section 3.4. We recall here that an illustration of these two error criterion is given in Figure 4. The first part of this section aims at validating validate the algorithm using one classical simulated data set: we observe here the influence of the reference sources positions. In a second part of this section, we investigate the noise propagation and the influence of observation's parameters such as the number of reference sources in the field or the number of frames available. In sections 4.1 to 4.2 , the number of distortion modes introduced is equal to the number of distortion mode searched in the data : $N_{\text {modes }}=N_{\text {modes, search }}$ while in the section 4.3 which is dedicated to the study of the aliasing effect, we consider $N_{\text {modes }} \geq N_{\text {modes, search. }}$. The settings used for each simulation are summarized in Table 1 .

\subsection{Validation of the algorithm}

This first simulation presented here aims to illustrate the validation the algorithm using one classical simulated data set. We consider

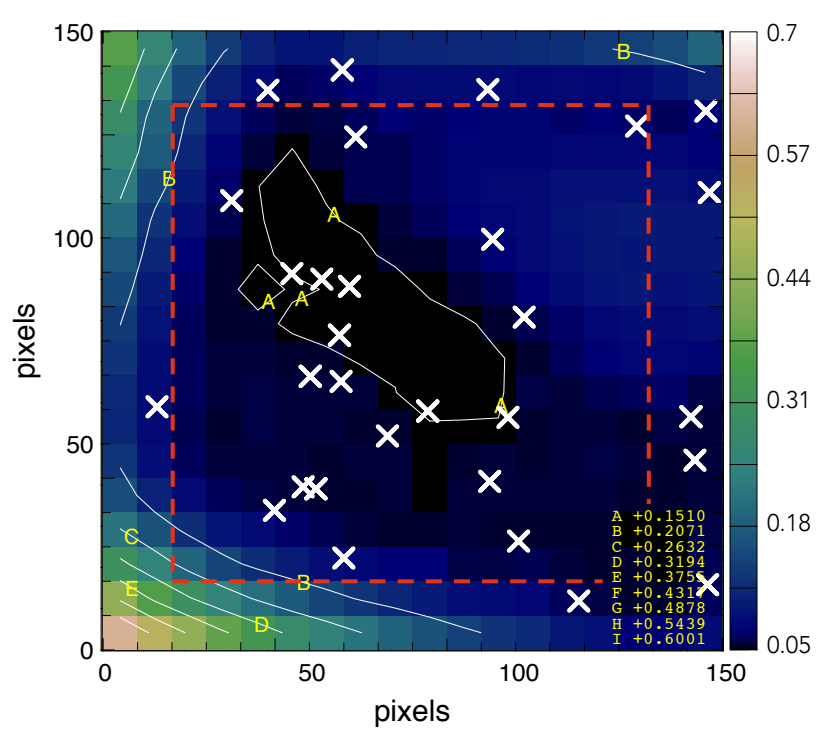

Figure 6. Distribution of the error $\sigma_{j^{\prime}}$ in the field. The error is calculated on a regular $150 \times 150$ grid of star. The white crosses represent the positions of the reference stars used in the minimization, $\left[X^{r e f}\right]_{1<j \leq N_{\text {star }}}$. Max is white $(\sim 0.7$ pixels $)$ and min is black $(\sim 0.05$ pixels $)$. The averaged value inside the red contour) is $\sigma_{\text {contour }}=0.16$ pixels (dark blue). Simulation runs with $N i m=10, N_{\text {star }}=30$, and $\sigma_{\text {meas }}=0.14$ pixels.

here 30 reference sources in the field and 10 frames $\left(N_{\text {star }}=30\right.$ and $\left.N_{i m}=10\right)$. Ten distortions modes are introduced on both the $x$-axis and the $y$-axis (i.e., $N_{\text {modes }}=10$, and $d=3$ ) and the level of noise considered is $\sigma_{\text {meas }}=0.14 \mathrm{px}$ (it corresponds to the faintest stars that can be detected with the GeMS/GSAOI system in a $60 \mathrm{~s}$ exposure : $K_{\mathrm{S}}$ magnitude $=18$ ). This simulation is referred to as Simulation 1 in Table 2.

Using these parameters and this pessimist level of noise, the precision obtained on the estimation of the reference positions is $b=0.05$ pixel which corresponds to 1 mas on GeMS data.

The standard deviation of the corrected position, $\sigma_{j^{\prime}}$ is calculated on a of grid of $150 \times 150$ stars (i.e. $0 \leq j^{\prime} \leq 150 \times 150$ ) regularly distributed in the image. The results are shown in Figure 4.1. Black corresponds to an error of $\sigma_{j^{\prime}}=0.05$ pixels and white corresponds to a value of $\sigma_{j^{\prime}}=0.7$ pixels. On this Figure, white crosses show the positions of the reference sources used in the minimization. As one might expect, the performance dramatically drops on the border of the field as no reference-source is constraining the model. The standard deviation of the corrected position averaged on the whole grid is $\sigma_{\text {grid }}=0.2$ pixels, while the averaged value calculated inside the square containing most of the stars used in the minimization (i.e., inside the red contour) is $\sigma_{\text {contour }}=0.16$ pixels (dark blue). In the following, the standard deviation, $\sigma$ is measured on a regular grid of $150 \times 150$ stars included in a given square containing most of the reference stars used in the minimization (i.e., in this particular simulation, inside the red contour represented in Figure 4.1):

\subsection{Noise propagation}

We now assess the noise propagation in the minimization using the same configuration: $N b_{\text {star }}=30$ and $N_{i m}=10$. Simulations are carried out for different levels of noise :

$\sigma_{\text {meas }} \in[0,0.03,0.07,0.14,0.28]$ pixels, and for each level of noise $n=100$ outcomes are computed. The two error criteria described 


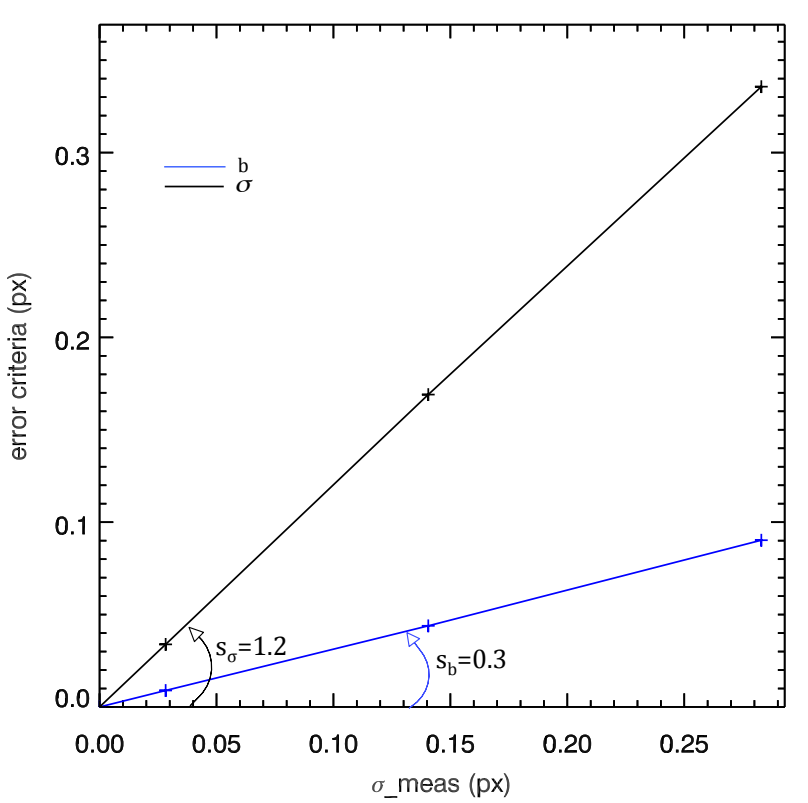

Figure 7. Noise propagation on the two error criterion. In black, $\sigma$ is the standard deviation of the corrected positions. In blue, $b$ is the error on the estimation of the reference. Both are shown versus the standard deviation of the noise in the star position measurements, $\sigma_{\text {me as }}$. For each level of noise, $n=100$ outcomes are computed, the error criterion are then quadratically averaged on the $n$ outcomes. The slopes of the noise propagation curves are noted $s_{\sigma}$ and $s_{b}$, respectively for the standard deviation of the corrected positions slope and the error on the reference estimation slope. Simulation runs with $N i m=10$ and $N_{\text {star }}=30$. For $\sigma_{\text {meas }}=0.14$ pixels, we found back the results of Section 4.1: $\sigma=0.16$ pixels and $b=0.05$ pixels.

previously are quadratically averaged on the $n$ outcomes. This study is referred as Simulation 2 in Table 2. The results is shown in Figure 7: the error on the estimation of the reference position, $b$ is plotted in blue line and the standard deviation of the corrected position $\sigma$, is plotted in black line. Both are plotted versus the standard deviation of the noise in the positions measurement of the data, $\sigma_{\text {meas }}$.

We find again the results derive for $\sigma_{\text {meas }}=0.14$ pixels (pessimist case) in the previous section and observe that the noise propagation on the two error criteria follows a linear comportement which slopes are noted $s_{b}$ and $s_{\sigma}$ hereafter (respectively for the slope of the noise propagation on the reference position estimation and for the slope of the noise propagation on the corrected position standard deviation). These slopes correspond to the amplification of the noise, which depends on intrinsic properties of the observation set, such as the number of reference sources and the number of frames available and used in the minimization. We detail both influences in the next sections.

\subsubsection{Impact of the number of frames}

In this section, we investigate the influence of the number of frames available on the noise propagation. Simulations as described previously are run with $N_{\text {star }}=30$ and a varying number of frames $N_{i m} \in[2,10,50,100]$. Then, the same error criteria are calculated (simulations referred as Simulation 3 in Table 2). The noise propa-

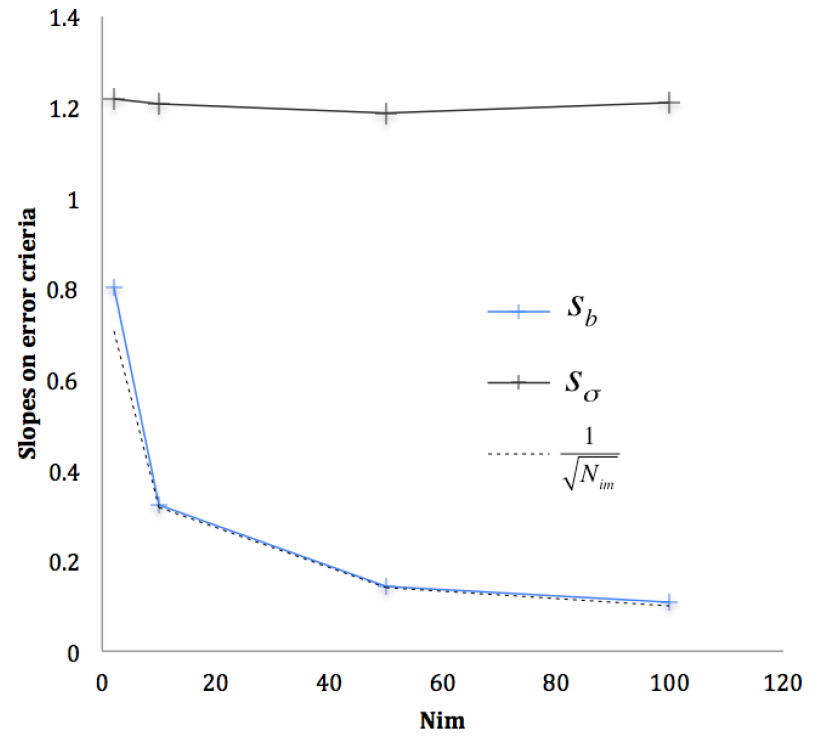

Figure 8. Influence of the number of frame. Black curve is the standard deviation of the corrected positions slope $\left(s_{\sigma}\right)$, and blue curve is the estimation of the reference error slope $\left(s_{b}\right)$. The curve $y=1 / \sqrt{x}$ is shown in grey dotted line. Simulation runs with $N_{\text {star }}=30$. For Nim $=10$, we found back the results of Section 4.2: $s_{\sigma}=1.2$ pixels and $s_{b}=0.3$ pixels.

gation still follows a linear behavior but this time, we are interesting in the slope of this linear propagation. Figure 8 shows the evolution of the error propagation slope of both error criteria, as the number of frames increases. The black curve shows the noise propagation on the corrected positions standard deviation slope, $s_{\sigma}$ as function of the number of frames. and blue curve shows the noise propagation on the reference position estimation slope, $s_{b}$ versus the number of frames. The curves show that the standard deviation of the corrected positions is independent of the number of frames. On the contrary, the error on the reference position estimation decreases when increases the number of frames. More precisely, we find that within a good approximation, the estimation of the reference precision improves as the square roots $N_{i m}$ :

$b \sim \frac{\sigma_{\text {meas }}}{\sqrt{N_{\text {im }}}}$

This is an expected behavior, as increasing the number of frames, increases the number of independent measurements while keeping the number of unknowns (star's reference positions) constant.

\subsubsection{Impact of the number of reference sources}

In this section we examine the influence of the number of reference sources used in the minimization following the same process as in the previous section. This time, the number of frame is fixed to $N_{i m}=10$ while the number of star is varying: $N_{\text {star }} \in[20,30,100,200]$. This simulation is referred as Simulation 4 in Table 2. The results plotted in Figure 9 show the slopes of the noise propagation on the two criteria versus the number of stars. The black curve shows the noise propagation on the corrected position's standard deviation slope $s_{\sigma}$, and the blue curve shows the noise propagation on the reference estimation slope $s_{b}$. Contrary to the number of frames, the number of reference sources affects only the standard deviation of the corrected frame $\sigma$, while the estimation of the reference positions error, $b$ is not affected. This 


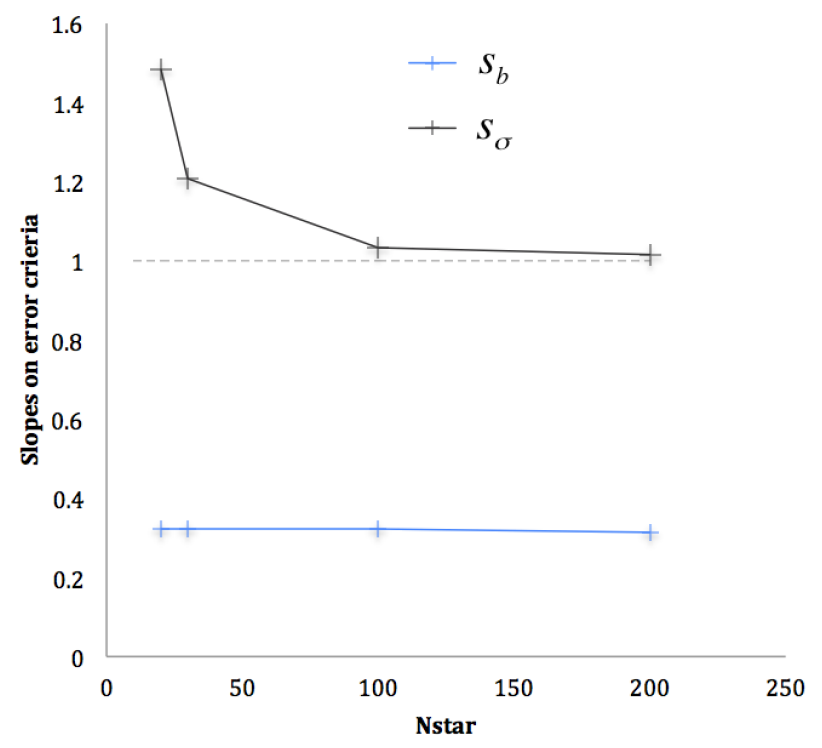

Figure 9. Influence of the number of reference sources used in the minimization. Black curve is the slope on the standard deviation of the corrected positions $\left(s_{\sigma}\right)$, and blue curve is the slope on the estimation of the reference error $\left(s_{b}\right)$. The curve $y=1$ is shown in grey dotted line. Simulation runs with $N i m=10$. For $N_{\text {star }}=30$, we found back the results of Sections 4.2 and 4.2.1: $s_{\sigma}=1.2$ pixels and $s_{b}=0.3$ pixels.

resulted from the fact that a higher number of reference sources allows a better estimation of the distortion in the whole field. In practice, with a hundred of reference sources available, the fundamental astrometric limitation mentioned in Section 3.4 is reached: $\sigma \simeq \sigma_{\text {meas }}$ which corresponds to $s_{\sigma} \simeq 1$.

In short, these results can be interpreted in the following way: the good estimation of a star position requires a large number of frames, i.e., a large number of measurements of this star position in the data. On the contrary, the ability to place every star in a same relative reference, or in other words, the ability to stack the images, is only governed by the number of reference sources available in the image to correctly estimate the distortion in the whole filed.

\subsection{Aliasing effects}

In the previous simulations we supposed: $N_{\text {modes }}=N_{\text {modes, search }}$. As aliasing effect will appear if the real number of distortion modes present in the data is higher than the number of modes taken into account in the model. When this happens, the non estimated modes are affecting the estimation of the other modes. To quantify this effect, we run simulations looking for $N_{\text {mode, search }}$ distortion modes, while we included $N_{\text {modes }} \geq N_{\text {modes, search modes in the data. We }}$ then look at the error on each estimated coefficient. The simulations are run with noiseless data, 10 frames and a high number of stars $\left(N_{\text {star }}=1000\right)$ to average the aliasing effect in the whole field. In a first approach, 3rd and lower polynomial orders are estimated $\left(N_{\text {modes, }}\right.$ search $\left.=10\right)$ while one additional 4 th order distortion mode $\left(b_{11}\right.$, proportional to $\left.\sim x^{4}\right)$ is introduced on the $x$-axis. This simulation is referred to as Simulation 5 in Table 2. The errors on each coefficient are shown in Figure. 10: Green bars represent the errors on the estimated coefficients quadratically averaged on all the images in absolute value, while red bars shows the value of the ad-

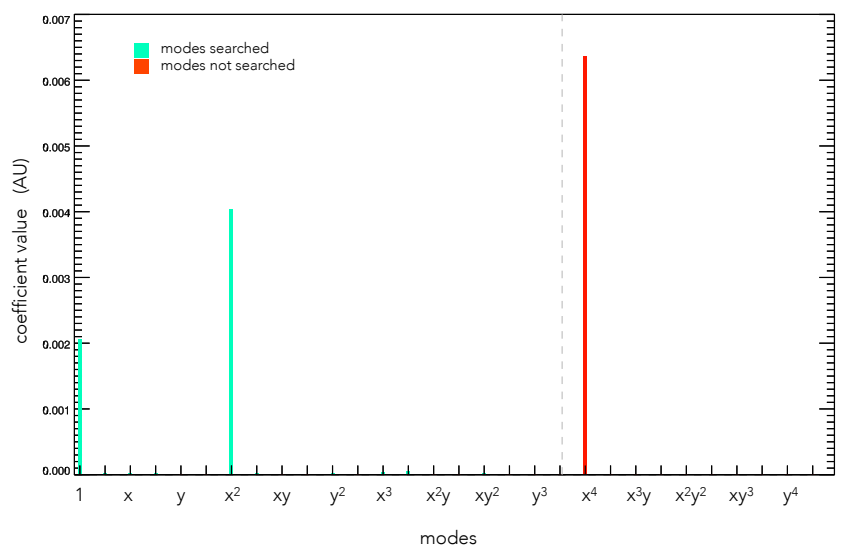

Figure 10. Aliasing of the $m=11$ mode proportional to $x^{4}$ on the $3^{\text {rd }}$ and lowers order modes. Green bars represent the errors on the estimated coefficients. Red bar represent the value of the additional non-estimated coefficient. For each mode, the first value corresponds to the $x$-axis distortion and the second value corresponds to the $y$-axis distortion. Each mode is identified according to their order in $x$ and $y$ variables.

ditional coefficient introduced and non-estimated. For convenience, the modes are identified according to their order in $x$ and $y$ variables. For each mode, the first value corresponds to the $x$-axis distortion and the second value corresponds to the $y$-axis distortion.

We observe here three important results about the aliasing of mode $b_{11}$, proportional to $\sim x^{4}$ and introduced on the $x$-axis: on average, affected coefficients are all coefficients associated to modes introduced on the $x$-axis. More precisely, affected coefficients are associated to modes proportional to $x^{0}$ and $x^{2}$ (i.e., to modes of the same parity as the aliased mode). Finally, the root mean square of the errors match with the value of the additional mode's coefficient: the energy is conserved. Those results can be generalized to all distortion modes: the energy is always aliased on modes of similar geometry and the total amount of energy aliased is preserved, which means that there is no amplification of the aliasing noise. Such results can be used to optimize the algorithm performance: depending on the noise level and on an estimate of the total amount of distortions in the data, we can predict the aliasing error term. By adjusting the number of distortion modes searched, we are able to keep it below a given threshold. Finally, these results provide precious informations on the effect of different distortions orders, that can be used to better constrain future instrumental designs.

\section{APPLICATION TO GEMS DATA}

In this section, we show the first application of the proposed method on on-sky data. The data used are collected with the Gemini MCAO instrument, GeMS combined with the Infra-Red (IR) camera GSAOI (for Gemini South Adaptive Optics Imager). The Gemini MCAO instrument GeMS is the first multi-Laser Guide Star (LGS) operational system on sky. It has been implemented on the Gemini South telescope and commissioned in 2013. It operates using two deformable mirrors conjugated at 0 and $9 \mathrm{~km}$ and a sodium-based LGS constellation composed of five spots: four are located at the corners of a 60 arcsec square, with the fifth positioned in the center. GeMS, as a facility instrument, can direct its light output to different science instruments installed at the Cassegrain focus of the Gemini South telescope. Combined with the IR camera GSAOI, it delivers 
Table 1. Summary table of detailed settings used in Simulations 1 to $5 . N_{\text {im }}$ is the number of frame, $N_{\text {star }}$ is the number of reference sources, $N_{\text {modes }}$ is

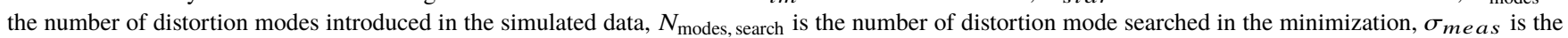
standard deviation of the Gaussian noise introduced in the position measurements and $n$ the number of outcomes computed for each level of noise.

\begin{tabular}{ccccccc} 
Simulation index & $N_{\text {im }}$ & $N_{\text {star }}$ & $N_{\text {modes }}$ & $N_{\text {modes, search }}$ & $\sigma_{\text {meas }}(\mathrm{px})$ & $n$ \\
\hline 1 & 10 & 30 & 10 & 10 & 0.14 & 1 \\
\hline 2 & 10 & 30 & 10 & 10 & {$[0,0.03,0.07,0.14,0.28]$} & 100 \\
\hline 3 & {$[2,10,50,100]$} & 30 & 10 & 10 & {$[0,0.03,0.07,0.14,0.28]$} & 100 \\
\hline 4 & 10 & {$[20,30,100,200]$} & 10 & 10 & {$[0,0.03,0.07,0.14,0.28]$} & 100 \\
\hline 5 & 10 & 1000 & 11 & 10 & 0.0 & 1 \\
\hline
\end{tabular}

near-diffraction limited images at Near-Infrared (NIR) wavelengths (from 0.9 to $2.4 \mu \mathrm{m}$ ) over a FoV of $85^{\prime \prime} \times 85^{\prime \prime}$. More details about the GeMS/GSAOI systems and its commissioning results are described in details in previous papers (see McGregor et al. (2004), Carrasco et al. (2012), d'Orgeville et al. (2012), Neichel et al. (2014a) and Rigaut et al. (2014)).

In the past few years, the GeMS/GSAOI systems has shown its ability to reach both good astrometric and good photometric precisions. Among the previous studies, we mention here two noteworthy results: Neichel et al. (2014b) show that for single-epoch, un-dithered data, an astrometric error below 0.2 mas can be achieved for exposure times exceeding one minute, and Turri et al. (2015) reached the deepest $K_{\mathrm{S}}$ photometry in a crowded field ever obtained from the ground with a magnitude error of 0.15 at $K_{\mathrm{S}}=22$ for a total exposure of $1920 \mathrm{~s}$.

However, regarding to multi-epoch or dithered data, the performance of the GeMS/GSAOI system has been confronted to difficulties due to the presence of static and variable distortions. The origins of these distortions are not totally understood but probable causes have been identified. First, the off-axis parabola present in the $\mathrm{AO}$ bench as well as the deformable mirror conjugated in altitude may introduce low spatial frequency orders of distortions (first and second orders). Then, from the analysis carried in Neichel et al. (2014c), we know that high spatial frequency are also present (around 15 degrees of freedom). Part of these high order distortions may be introduced in dithered data by the gaps between the four GSAOI's detectors. Finally, observational factors such as the Natural Guide Stars (NGS) constellation and the telescope pointing that induces gravity flexure and movement of the AO-bench due to the Cassegrain configuration of the telescope might introduce variable terms of distortions.

GeMS/GSAOI distortions have recently been calibrated by Massari et al. (2016) who used HST data as an external distortionfree reference. The target field is the globular cluster NGC6681, which was observed with both systems: WFC/ACS of HST in 2006, as part of the GO-10775 program (PI : Sarajedini), and GeMS/GSAOI in 2013 as part of the programs GS-2012B-SV-406, GS-2013A-Q-16 and GS-2013B-Q-55 (PI : McConnachie). They derived from the HST observations the positions of 7770 stars that were then moved according to their Proper Motion (e.g. Massari et al. (2013)) to construct a distortion-free reference frame at the epoch of the GeMS dataset, 6.914 years later.

Their processed distortion map, including second and higher distortion orders, shows a peak values of 5 pixels ( 100 mas), on the $x$-component while the $y$-component range below 0.5 pixels (10 mas). In addition, circular structures are seen at the center of each chip, where the $x$-component is minimal and then, changes its direction as shown in Figure 11. This distortion solution is derived

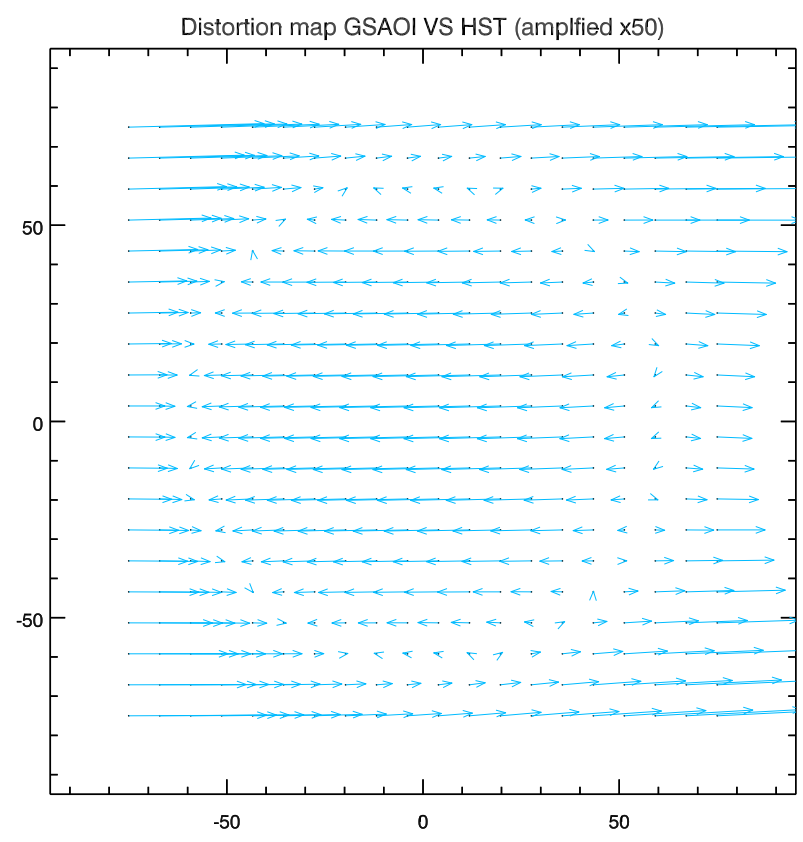

Figure 11. GeMS/GSAOI distortion map. The blue arrow show the displacement induced by second and higher distortion orders on one detector. The displacements are amplified by 50 .

for the specific data set of the NGC6681 cluster and can only be applied to this observation set due to the variability of the distortions. However, it represents the best estimation of the GeMS/GSAOI distortion currently available. It is thus used in the following, as input parameter in the method and mentioned as static distortion map.

Based on this static distortion map associated to the GeMS/GSAOI instrument, we perform in this Section the first application on real data of the new distortion correction method proposed in this paper. This Section is organized as follows: in a first part, we briefly describe the observations set and data reduction. In a second part, we detail the construction of the star position catalogs. The third part describes the distortion correction settings and the last part shows the results and highlights the gain brought by this method.

\subsection{Observation and data reduction}

The distortion correction is performed on a GeMS/GSAOI data set extracted from a recent observation of a very active and young star- 
forming region named $\mathrm{N} 159 \mathrm{~W}$ and located in the Large Magellanic Cloud (LMC). This region was previously observed by Bernard et al. (2016), Deharveng et al. (1992), Testor et al. (2007), Chen et al. (2010) in order to study the properties of the cluster stellar members and bring new elements to our understanding of the massive star formation process. In this study, we use the 11 frames obtained in $K_{\mathrm{S}}$ filter. The $N 159 \mathrm{~W}$ field provides a large number of well-isolated stars and is therefore an good case to experiment new methods of distortion correction. The data were obtained during the night of December 8th 2014 as part of program GS-2014B-C-2 (P.I. B. Neichel). Each observation consists of one science field dithered randomly by a $5^{\prime \prime}$ rms shift to remove gaps between the detectors. The averaged FWHM in $K_{\mathrm{S}}$ band is 90 mas, while the averaged Strehl ratio (SR) is $14 \%$. The coordinates of the center of the field are RA $=05 \mathrm{~h} 39 \mathrm{~m} 40 \mathrm{~s} ; \mathrm{DEC}=-69^{\circ} 45^{\prime} 55^{\prime \prime}$.

The raw images were processed by subtracting dark frames and removing bad pixels from the analysis. Flat-field calibration was performed using twilight sky flats. Sky subtraction was accomplished by forming the median of the dithered frames taken outside of the cluster and subtracting this median from each exposure. As we previously mentioned, the GSAOI camera is composed of 4 chips separated by gaps. In the following, we aim to correct distortion of data collected with the lower left detector as we only have available static distortion for this one chip. Hereafter, it is referred to as detector 2 .

\subsection{Construction of input catalogs}

To perform the distortion correction, a list of stellar positions and brightnesses in each exposure is needed, as well as the measurement error $\sigma_{\text {meas, } j}$ associated to each star $j$. The measurement error will be used for both, the estimation (as it is related to the weighted coefficient $w$ as described in Section 5.3) and the quantification of the algorithm performance in Section 5.3.1. The first part of this section is dedicated to the position and flux measurements process. The second part describe the simulations used to estimate the measurement errors.

\subsubsection{Position measurements}

The stellar positions and brightness catalog is generated using a combination of two star detection tools. Sextractor (Bertin \& Arnouts (1996)) is first used to perform a fast detection of the objects in the field and derive position measurements. However, as Sextractor has not been designed to perform accurate astrometry, we use in a second phase a home-made fitting method developed with the interpreted-language Yorick (Munro (1995)), to measure accurate stellar positions. We fit the star intensity distribution using a Moffat profile defined as follows:

$I=I_{0} *\left[1+(X / d x)^{2}+(Y / d y)^{2}\right]^{-\beta}+I_{b k g}$

where $X=\left(x-x_{0}\right) \cos \theta+\left(y-y_{0}\right) \sin \theta$ and $Y=$ $\left(y-y_{0}\right) \cos \theta-\left(x-x_{0}\right) \sin \theta$. The free parameters of the fit are the positions $\left(x_{0}\right.$ and $\left.y_{0}\right)$, the intensity, $\left(I_{0}\right)$, the width in both directions ( $d x$ and $d y)$, the position angle $(\theta)$, and the beta index $(\beta)$. The background $\left(I_{b k g}\right)$ is fitted simultaneously.

As we need to identify stars positions in each frame, we only consider relatively well-isolated stars in order to avoid any confusion. In addition, any star that did not appear in at least 4 exposures is discarded. This gives us a stellar catalog with positions and flux

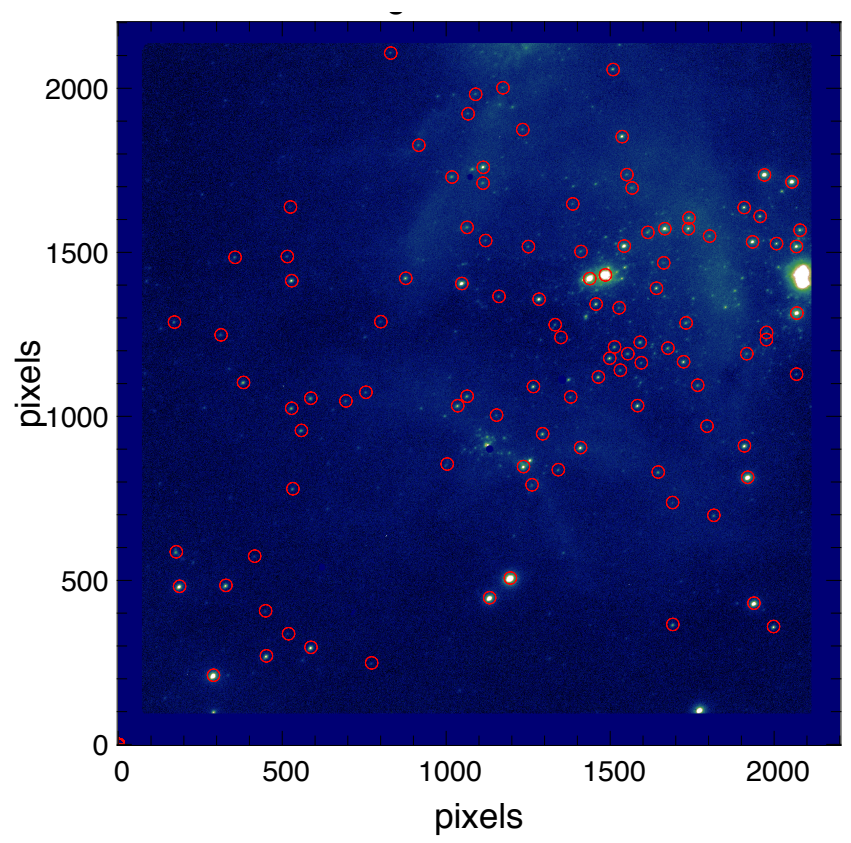

Figure 12. One typical reduced frame imaged with the detector 2. Stars used in the minimization are red circled.

measurements for each frame. The final number of stars in each catalog range between and 61 and 78. Figure 12 shows the lower left part of one reduced frame, corresponding to the part imaged with the detector 2 . Stars referenced in the catalog are marked by a red circle.

\subsubsection{Estimation of noise measurements}

To estimate the noise measurement, in the specific case of the N159W observations, we use simulations. The idea is to simulate and ideal Moffat PSF and to embed it, at a known position, in a background image derived from the $N 159 \mathrm{~W} K_{\mathrm{S}}$-band data. Then the position is estimated again using the position measurement process described in the previous section (5.2.1). The measurement error appears immediately as the distance between the real position of the PSF and the fitted position of the PSF. The detector noise is already included in the background and the photon noise is added using a Poisson distribution. Different PSF flux levels are explored by scaling the PSF before the photon-noise computation and for each PSF flux level, we simulated a set of 100 images with different known positions of the PSF. The positional uncertainties are then quadratically averaged on the 100 positions for each intensity level. Results are presented in Figure 13. The solid lines shows the astrometric error (in pixels) versus the flux (in Analog-to-Digital Units (ADU)). The four curves corresponds to four different Full Width Half Maximum (FWHM) of the simulated PSF: 70, 90, 110 and 130 mas ( respectively in black, orange, blue and red line). The grey dashed line shows the $1 /$ Flux slope and the grey dotted line shows the $1 / \sqrt{\text { Flux }}$ slope. These two slopes highlights two regimes: a $1 /$ Flux evolution for fluxes lower than $10^{6}$ ADU and a $1 / \sqrt{\text { Flux }}$ for higher fluxes. The former regime is dominated by the detector and sky noise, the latter being dominated by the PSF photon noise. 


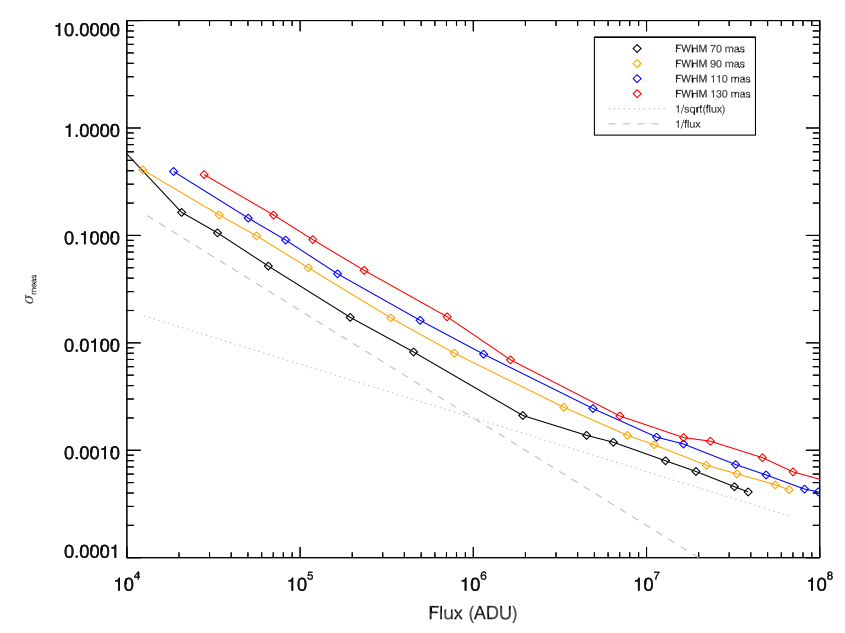

Figure 13. Astrometric error (in pixels) versus the flux (in ADU) in specific N159W data for different Full Width Half Maximums: 70 mas (in black), 90 mas (in orange), 110 mas (in blue) and 130 mas in red. The grey dashed line shows the $1 /$ Flux slope and the grey dotted line shows the $1 / \sqrt{\text { Flux }}$ slope.

\subsection{Distortion correction}

The distortion correction is then performed using the following settings (summarized in Table 2): The weighting coefficient $w_{i, j}$ is set for each star $j$ according to its positional uncertainty $\sigma_{\text {meas }, j}$. The positional uncertainty is derived from the flux of each star according to the simulation curve presented in the last section for FWHM $=90$ mas, which is the averaged FWHM in the data. It ranges between 0.002 pixels and 0.14 pixels (respectively 0.02 mas and 2.8 mas), mostly depending on the magnitude of the objects.

$w_{i, j}=\frac{1}{\sigma_{\text {meas }, j}^{2}}$

We recall here that the distorsion map derived for the specific data set of NGC6681 by Massari et al. (2016) is the best estimation of the static distorsion present in the GeMS/GSAOI instrument currently available. The initial distortion coefficients $[A]^{0}$ are then derived from this static distortion solution fitted on the 2D Legendre polynomials normed basis. As the fit does not improve beyond 10 distortion modes considered on both $x$ and $y$ axis, we use this setting to perform the distortion correction: $N_{\text {mode,search }}=10$ which corresponds to 3 rd order polynomials $(d=3)$.

\subsubsection{Results}

The distortion solution derived for each frame is varying around the distortion solution provided by Massari et al. (2016). For exemple, Figure 14 shows the estimated distortion coefficient value $A_{i_{0}}$ of frame $i_{0}$ (in blue bars) and the distortion coefficients derived from the static distortion solution, $[A]^{0}$ (in orange bars).

In order to estimate the correction efficiency we plot the corrected position standard deviation of each star $\left(\sigma_{j}\right)$ as function of its flux. Each star is represented by a green cross on Figure. 15. As a comparison, we also plot the same quantities obtained by performing a simple shift correction (black), by performing a correction of the static distortion in addition to the shift correction (blue), and by performing a relative distorsion correction as it was originally done

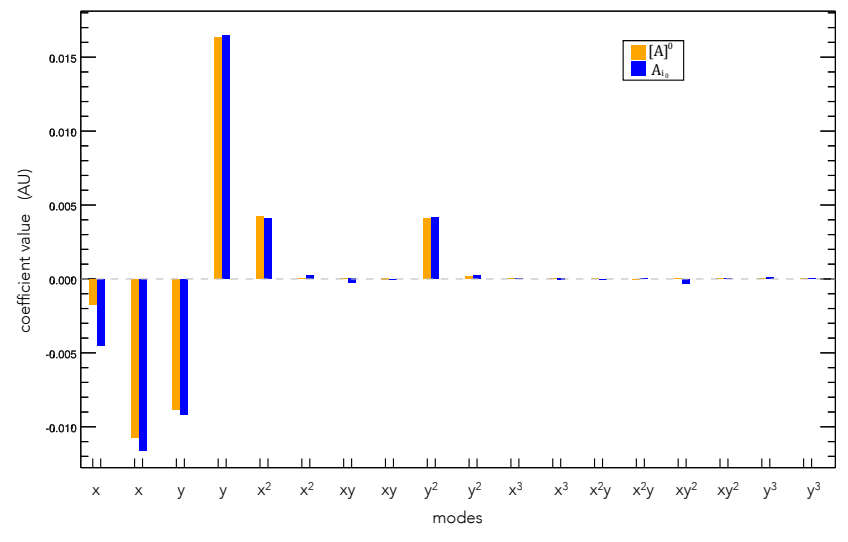

Figure 14. Bargraph of the estimated distortion coefficient $A_{i_{0}}$ associated to frame $i_{0}$ (in blue bars) and the distortion coefficients associated to the static distortion solution, $[A]^{0}$ (in orange bars). The modes are identified according to their order in $x$ and $y$ variables. For each mode, the first value corresponds to the $x$-axis distortion and the second value corresponds to the $y$-axis distortion.

in Bernard et al. (2016) using the same data set (red). The static distortion is removed by applying to each frame the static distortion map derived by Massari et al. (2016) and the relative distorsion correction is applied following the process described in detail in Bernard et al. (2016). On Figure 15, the curves shows the position uncertainty as function of the flux for a 90 mas FWHM star (orange line) and a 110 mas FWHM star (blue line). Those are the curves calculated in Section 5.2.1 specifically for the $N 159 \mathrm{~W}$ data.

The Figure 15 illustrates the gain brought by the different methods : the static distortion correction improves the astrometric precision to an averaged $\sigma_{\text {static disto. }}=0.41$ pixels and a standard deviation of 0.21 pixels, compare to a simple shift correction where $\sigma_{\text {shift }}=0.83$ pixels and the standard deviation is 0.4 pixels. However, the static distortion map derived for the specific data set of the NGC6681 cluster is not perfectly suited for any other set of observation due to the variability of the distortions. By applying a relative correction of distortion, these variabilities are taken into account as each frame is corrected individually according to one reference frame chosen arbitrary within the data. The astrometric error then falls down to an averaged precision of $\sigma_{\text {new meth. }}=0.24$ pixels, and a standard deviation of 0.21 pixels.

The method proposed in this paper presents both advantages to handles the distorsion variabilities by adapting distortion maps to each set of observations and each individual frame, and to provide an optimal noise propagation thanks to the weighted coefficient. In this way, the error term due to distortion is minimized and the astrometric performance is further improved to an averaged precision of $\sigma_{\text {new meth. }}=0.20$ pixels, and a standard deviation of 0.20 pixels. Plus, we recall here that an additional advantage of the proposed method is to provide an estimation of the distortion-free reference. In the case of a perfect optical system, the astrometric error is determined by the measurement noise. This means that any source of additional error needs to be compensated down to this level if we want to fully exploit the data capabilities. That is what we achieve by performing the method presented in this paper: the distortion error term is minimized and the resulting astrometric uncertainties are dominated by noise measurement. Of course, the final astrometric performance still depends on a large panel of parameters, such as the seeing conditions, the FWHM, the nature of the field : crowded 
Table 2. Summary table of settings used in distortion correction of $N 159 \mathrm{~W}$ data. $N_{i m}$ is the number of frame, $N_{\text {st ar }}$ is the number of reference sources, $N_{\text {modes, search }}$ is the number of distortion mode searched in the minimization, $w$ is the weight coefficient and [A $]^{0}$ the initialization of the distortion coefficients set according to the prior knowledge of the static distortion

\begin{tabular}{cccccc}
$N_{\text {im }}$ & $N_{\text {star }}$ & $N_{\text {modes, search }}$ & $\mathrm{d}$ & $w$ & {$[A]^{0}$} \\
\hline 11 & {$[61: 78]$} & 10 & 3 & $1 / \sigma_{\text {meas }}^{2}$ & $\begin{array}{c}\text { Massari et al. (2016) static distortion solution } \\
\text { fitted on 2D Legendre normed basis }\end{array}$ \\
\hline
\end{tabular}

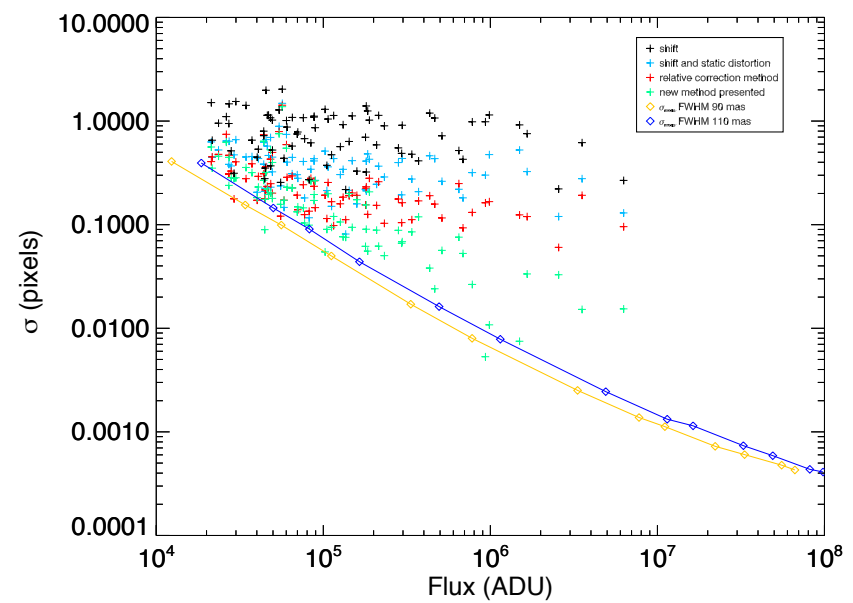

Figure 15. Corrected position standard deviation of each star $\left(\sigma_{j}\right.$ in pixel) as function of its flux (ADU) for different type of corrections: A shift correction (in black). A static distortion correction using the static distortion map and a shift correction (in blue), a relative distorsion correction (red), and a distortion correction performed with the method preposed (in green). Orange and blue curves represent the astrometric error versus the flux calibrated in GeMS/GSAOI data corresponding to a 90 mas and 110 mas FWHM respectively.

or sparse, the presence of background structures, etc. However, the proposed method guaranties an optimal minimization of the distortion error term. Plus, the weighted property of the minimization provides an ideal noise management: this allows the use of all reference sources available, even the faintest ones, which is an advantage for sparse field studies.

As a final step, the correction is applied to each of the 11 frames by an interpolation process using the estimated distortion coefficient $[\widehat{A}]$. The final stacked image is shown in Figure 16. Parts of the field (indicated by a white square) are zoomed in and shown in Figure 17. This Figure shows results obtained by performing the optimal distortion correction presented (left panel) and a static distortion correction (right panel). Note that the brightest regions are saturated in order to better show the resolution improvement brought by our method on the faintest stars.

\section{CONCLUSION}

In this paper we presented an new and optimal method of distortion correction for high angular resolution images. Based on a priorknowledge of the static distortion present in the data, this method aims to correct the dynamical distortions by specifically adapting to each set of observation and each frame. The method consists in an inverse problem approach solved by a Weighted Least Square minimisation. Thanks to the weighted property of the minimization, we guaranty a minimal noise propagation that allows the use

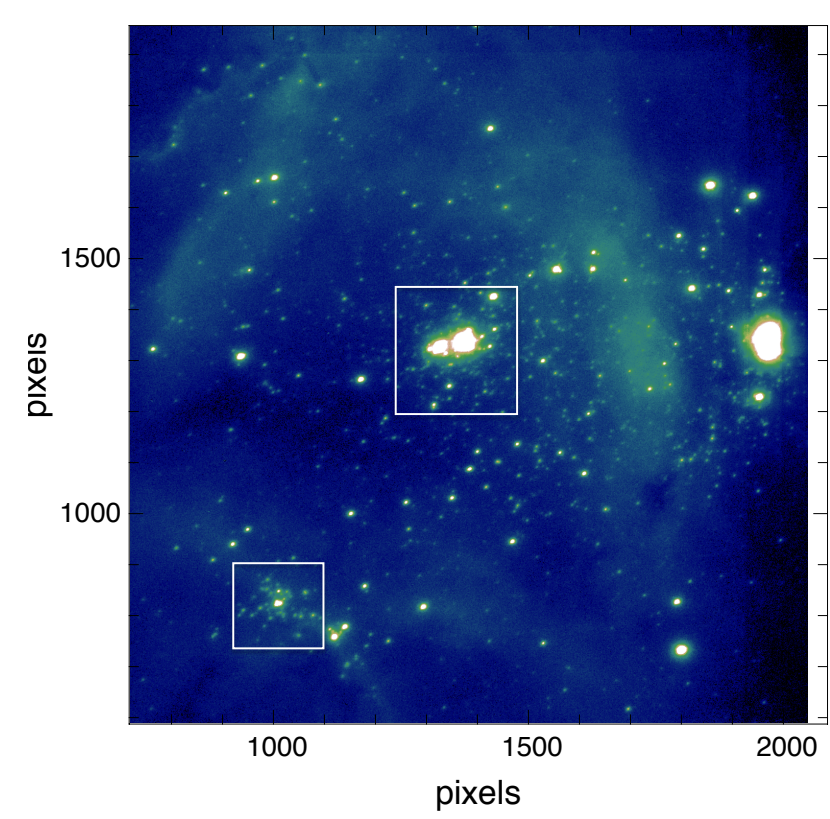

Figure 16. Final stack on the 11 frames individually corrected from distortion by performing the optimal distortion correction presented. White squares show parts of the fields that are zoomed in and shown in Figure 17.

of the faintest reference sources as algorithm input. This is a very valuable advantage if working on sparse fields images. The resolution provides an estimation of both a distortion-free reference and a distortion solution associated to each frame. Depending on the scientific aim of the study (astrometry, photometry), both parameters can be used independently. We presented the complete algorithm implementation as well as simulations studies aiming to characterize the method performance facing different type of observations: crowded or sparse field, varying number of frame, positions of the reference sources, number of distortion modes. Those results allow us to optimize the algorithm settings regarding each configuration of data and scientific goal, and to predict the expected performance for a given observation set. Finally, we show the first application of the method using on-sky data collected with the Gemini MCAO instrument, GeMS. We reach an astrometric precision of 0.2 pixels which represents a gain of factor two compared to a classical static distortion correction and a factor 1.2 compare to a relative distorsion correction. The final astrometric performance still depends on a large number of parameters, such as the seeing conditions, the FWHM, the nature of the field : crowded or sparse or the presence of background structures. However, the proposed method guaranties an optimal minimization of the distortion error term which conducts to a resulting astrometric error falling down to the noise measurement level. 

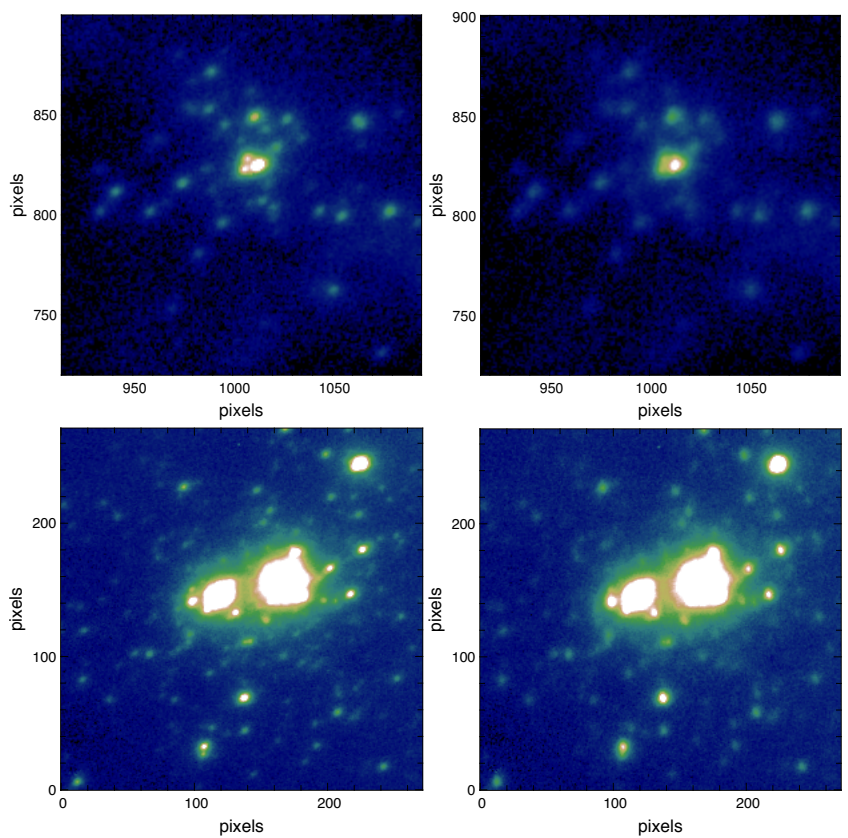

Figure 17. Part of the final stack image obtained by performing the the optimal distortion correction presented (left panel) and a static distortion correction (right panel). Note that the brightest regions are saturated in order to better show the resolution improvement brought by our method on the faintest stars.

\section{ACKNOWLEDGEMENTS}

Based on observations obtained at the Gemini Observatory, which is operated by the Association of Universities for Research in Astronomy, Inc., under a cooperative agreement with the NSF on behalf of the Gemini partnership: the National Science Foundation (United States), the National Research Council (Canada), CONICYT (Chile), the Australian Research Council (Australia), Ministério da Ciência, Tecnologia e Inovação (Brazil) and Ministerio de Ciencia, Tecnologiaa e Innovación Productiva (Argentine).

B. Neichel and A. Bernard acknowledge the financial support from the French ANR program WASABI to carry out this work.

The authors are grateful to Dr Carrasco for careful reading of the paper and valuable suggestions and comments.

\section{REFERENCES}

Abuter R., Schreiber J., Eisenhauer F., Ott T., Horrobin M., Gillesen S., 2006, New Astron. Rev., 50, 398

Anderson J., King I. R., 2003, PASP, 115, 113

Anderson J., King I. R., 2004, Technical report, Multi-filter PSFs and Distortion Corrections for the HRC

Anderson J., Bedin L. R., Piotto G., Yadav R. S., Bellini A., 2006, A\&A, 454, 1029

Bellini a., Bedin L. R., 2009, PASP, 121, 20

Bellini A., Bedin L. R., Piotto G., Milone A. P., Marino A. F., Villanova S., 2010, AJ, 140, 631

Bernard A., Neichel B., Samal M. R., Zavagno A., Andersen M., Evans C. J., Plana H., Fusco T., 2016, A\&A, 592, A77

Bertin E., Arnouts S., 1996, A\&AS, 117, 393

Cameron P. B., Kulkarni S. R., 2007, in American Astronomical Society Meeting Abstracts. p. 996
Cameron P. B., Britton M. C., Kulkarni S. R., 2009, AJ, 137, 83

Carrasco E. R., et al., 2012, in Adaptive Optics Systems III. p. 84470N, doi: $10.1117 / 12.926240$

Chen C.-H. R., et al., 2010, ApJ, 721, 1206

Deharveng L., Caplan J., Lombard J., 1992, A\&AS, 94, 359

Dunkl C., Xu Y., 2014, Orthogonal Polynomials of Several Variables. Encyclopedia of Mathematics and its Applications, Cambridge University Press, https: //books.google.fr/books?id=eRdEBAAAQBA J

Fritz T., et al., 2010, MNRAS, 401, 1177

Ghez A. M., et al., 2008, ApJ, 689, 1044

Gratadour D., Mugnier L. M., Rouan D., 2005, A\&A, 443, 357

Hasan H., Bely P. Y., 1994, in Hanisch R. J., White R. L., eds, The Restoration of HST Images and Spectra - II. p. 157

Libralato M., Bellini A., Bedin L. R., Piotto G., Platais I., Kissler-Patig M., Milone A. P., 2014, A\&A, 563, A80

Lu J. R., Ghez A. M., Hornstein S. D., Morris M. R., Becklin E. E., Matthews K., 2009, ApJ, 690, 1463

Massari D., Bellini A., Ferraro F. R., van der Marel R. P., Anderson J., Dalessandro E., Lanzoni B., 2013, ApJ, 779, 81

Massari D., et al., 2016, A\&A, 595, L2

McGregor P., et al., 2004, in Moorwood A., Iye M., eds, Proc. SPIEVol. 5492, Ground-based Instrumentation for Astronomy. pp 1033-1044, doi: $10.1117 / 12.550288$

Meyer E., Kürster M., Arcidiacono C., Ragazzoni R., Rix H.-W., 2011, A\&A, 532, A16

Mugnier L., 2008, in Léna P., Rouan D., Lebrun F., Mignard F., Pelat D., eds, , L'observation en astrophysique. EDP Sciences, Les Ulis, France, Chapt. 9, section 6, pp 591-613

Mugnier L. M., Fusco T., Conan J.-M., 2004, Journal of the Optical Society of America. A, Optics, image science, and vision, 21, 1841

Munro D. H., 1995, Comput. Phys., 9, 609

Neichel B., et al., 2014a, Proc. AO4ELT3, pp 1-9

Neichel B., et al., 2014b, MNRAS, 440, 1002

Neichel B., Lu J. R., Rigaut F., Ammons S. M., Carrasco E. R., Lassalle E., 2014c, MNRAS, 445, 500

Reid M. J., Menten K. M., 2007, ApJ, 671, 2068

Rigaut F., et al., 2014, MNRAS, 437, 2361

Service M., Lu J. R., Campbell R., Sitarski B. N., Ghez A. M., Anderson J., 2016, PASP, 128, 095004

Testor G., Lemaire J. L., Kristensen L. E., Field D., Diana S., 2007, A\&A, 469,459

Trippe S., Davies R., Eisenhauer F., Schreiber N. M. F., Fritz T. K., Genzel R., 2010, MNRAS, 402, 1126

Turri P., McConnachie A. W., Stetson P. B., Fiorentino G., Andersen D. R., Véran J.-P., Bono G., 2015, ApJ, 811, L15

Ubeda L., Kozhurina-Platais V., 2013, Technical report, ACS/WFC Geometric Distortion: a time dependency study

Ye J., Gao Z., Wang S., Cheng J., Wang W., Sun W., 2014, J. Opt. Soc. Am. A, 31, 2304

Yelda S., Lu J. R., Ghez A. M., Clarkson W., Anderson J., Do T., Matthews K., 2010, ApJ, 725, 331

d'Orgeville C., et al., 2012, in Adaptive Optics Systems III. p. 84471Q, doi: $10.1117 / 12.925813$

This paper has been typeset from a $\mathrm{T}_{\mathrm{E}} \mathrm{X} / \mathrm{LAT}_{\mathrm{E}} \mathrm{X}$ file prepared by the author. 